\title{
On the Splitting of the Einstein Field Equations with respect to General $(1+3)$ Threading of Spacetime
}

\author{
Aurel Bejancu and Hani Reda Farran \\ Department of Mathematics, Kuwait University, P.O. Box 5969, 13060 Safat, Kuwait \\ Correspondence should be addressed to Hani Reda Farran; hani.farran@ku.edu.kw
}

Received 5 July 2016; Accepted 3 October 2016

Academic Editor: Eugen Radu

Copyright ( 2016 A. Bejancu and H. R. Farran. This is an open access article distributed under the Creative Commons Attribution License, which permits unrestricted use, distribution, and reproduction in any medium, provided the original work is properly cited.

Based on general $(1+3)$ threading of the spacetime $(M, g)$, we obtain a new and simple splitting of both the Einstein field equations (EFE) and the conservation laws in $(M, g)$. As an application, we obtain the splitting of EFE in an almost FLRW universe with energy-momentum tensor of a perfect fluid. In particular, we state the perturbation Friedmann equations in an almost FLRW universe.

\section{Introduction}

The present paper is a continuation of [1], wherein an new approach on the $(1+3)$ threading of spacetime with respect to an arbitrary timelike vector field has been developed. The study in [1] refers to Lorentz metrics given by (2a), (2b), and (2c) and subject to the condition that $\Phi$ is independent of the time coordinate. We remove this condition, and therefore the results are valid for any Lorentz metric of a spacetime. Another important issue of the present paper is that the whole study is developed in the general setting of a spacetime with a spatial distribution that is not necessarily integrable. The threading frames and coframes, the spatial tensor fields, and the Riemannian spatial connection are the main tools used throughout the paper. These geometric objects enable us to obtain new and simple splitting of EFE and to apply it to the structure of an almost FLRW universe. The new approach developed in this paper can be extended to threading of higher-dimensional universes. In this respect, we mention [2], wherein $(1+1+3)$ threading of a $5 D$ universe has been developed.

Now, we outline the content of the paper. In Section 2 we present the main geometric objects which constitute the foundation of a general $(1+3)$ threading formalism of a spacetime $(M, g)$ with respect to an arbitrary timelike vector field. We close this section with local expressions of the LeviCivita connection $\nabla$ in terms of spatial tensor field and of the local coefficients of the Riemannian connection $\bar{\nabla}$ (cf. (23a), (23b), (23c), and (23d)). In Section 3 we state, for the first time in the literature, the spatial Bianchi identities in the general case when the spatial distribution is not necessarily integrable (cf. (37), (38), and (39)). The structure equations on $(M, g)$ induced by the $(1+3)$ threading formalism are presented in Section 4 (cf. (47a), (47b), (47c), (47d), (48a), (48b), (48c), and (48d)). They play an important role in the next sections, wherein we relate tensor fields on $M$ with spatial tensor fields. In Section 5 we obtain simple expressions for the local components of the Ricci tensor of $(M, g)$ with respect to the threading frame field and for the scalar curvature (cf. (63a), (63b), (63c), (64a), (64b), (64c), (66a), and (69)). The splittings of both the Einstein gravitational tensor field and the energy-momentum tensor field are stated in Section 6 (cf. (73a), (73b), (73c), (81), (84), and (86)). In Section 7 we obtain the spatial, mixed, and temporal EFE (cf. (89), (90a), (90b), and (91)). Also, we state (93) which, in the particular case when the threading is taken with respect to a unit vector field, becomes the well-known Raychaudhuri-Ehlers equation. A new splitting of conservation laws with respect to general $(1+3)$ threading of spacetime is given in Section 8 (cf. (98), and (99)). Also, we compare our results with what is known in the literature on this matter. Finally, in Section 9 we apply the general theory developed in the paper to the $(1+3)$ threading of an almost FLRW universe. We close the paper with Conclusions and Appendices A, B, and C. 


\section{The $(1+3)$ Threading Formalism with respect to a Nonnormalized Timelike Vector Field}

Recently, a new approach on the $(1+3)$ threading of spacetime with respect to a nonnormalized timelike vector field has been developed (cf. [1]). In the most general setting that we explain in this section, we recall the main geometric objects introduced in [1]. Also, we introduce the extrinsic curvature tensor field for the spatial distribution and use it in the expressions of the Levi-Civita connection on a $4 D$ spacetime.

Let $(M, g)$ be a $4 D$ spacetime and $\xi$ be a timelike vector field on $M$ that is not necessarily normalized. Then, the tangent bundle TM of $M$ admits the decomposition

$$
\mathrm{TM}=\mathrm{VM} \oplus \mathrm{SM},
$$

where VM is the temporal distribution spanned by $\xi$ and SM is the spatial distribution that is complementarily orthogonal to VM in TM.

Throughout the paper we use the ranges of indices: $i, j, k, \ldots \in\{1,2,3\}$ and $a, b, c, \ldots \in\{0,1,2,3\}$. Also, for any vector bundle $E$ over $M$, denote by $\Gamma(E)$ the $\mathscr{F}(M)$-module of smooth sections of $E$, where $\mathscr{F}(M)$ is the algebra of smooth functions on $M$.

The congruence of curves that is tangent to VM determines a coordinate system $\left(x^{a}\right)$ on $M$ such that $\xi=\partial / \partial x^{0}$. Next, we put

$$
\begin{aligned}
\xi_{0} & =g\left(\frac{\partial}{\partial x^{0}}, \frac{\partial}{\partial x^{0}}\right)=-\Phi^{2}, \\
\xi_{i} & =g\left(\frac{\partial}{\partial x^{i}}, \frac{\partial}{\partial x^{0}}\right), \\
g_{i j} & =g\left(\frac{\partial}{\partial x^{i}}, \frac{\partial}{\partial x^{j}}\right),
\end{aligned}
$$

wherein $\Phi$ is a nonzero function on $M$.

Remark 1 . Note that, in [1], $\Phi$ was supposed to be independent of $x^{0}$. Here, we remove this condition on $\Phi$, and thus the results stated in the present paper are valid for any Lorentz metric $g$ on $M$.

In this approach we use the threading frame field $\left\{\partial / \partial x^{0}, \delta / \delta x^{i}\right\}$ and the threading coframe field $\left\{\delta x^{0}, d x^{i}\right\}$ defined as follows:

$$
\begin{aligned}
& \frac{\delta}{\delta x^{i}}=\frac{\partial}{\partial x^{i}}-A_{i} \frac{\partial}{\partial x^{0}} \\
& \delta x^{0}=d x^{0}+A_{i} d x^{i}
\end{aligned}
$$

where we put

$$
A_{i}=-\Phi^{-2} \xi_{i}
$$

The Lie brackets of the vector fields from the threading frame are given by

$$
\left[\frac{\delta}{\delta x^{j}}, \frac{\delta}{\delta x^{i}}\right]=2 \omega_{i j} \frac{\partial}{\partial x^{0}}
$$

$$
\left[\frac{\partial}{\partial x^{0}}, \frac{\delta}{\delta x^{i}}\right]=a_{i} \frac{\partial}{\partial x^{0}}
$$

where we set

$$
\begin{aligned}
\omega_{i j} & =\frac{1}{2}\left\{\frac{\delta A_{j}}{\delta x^{i}}-\frac{\delta A_{i}}{\delta x^{j}}\right\} \\
& =\Phi^{-2}\left\{c_{i} \xi_{j}-c_{j} \xi_{i}+\frac{1}{2}\left(\frac{\delta \xi_{i}}{\delta x^{j}}-\frac{\delta \xi_{j}}{\delta x^{i}}\right)\right\}, \\
c_{i} & =\Phi^{-1} \frac{\delta \Phi}{\delta x^{i}}, \\
a_{i} & =-\frac{\partial A_{i}}{\partial x^{0}}=\Phi^{-2}\left\{\frac{\partial \xi_{i}}{\partial x^{0}}-2 \Psi \xi_{i}\right\}, \\
\Psi & =\Phi^{-1} \frac{\partial \Phi}{\partial x^{0}} .
\end{aligned}
$$

Taking into account that the Levi-Civita connection $\nabla$ on $(M, g)$ is torsion-free, from (5a) we deduce that

$$
\omega_{i j}=\frac{1}{2} \Phi^{-2} g\left(\nabla_{\delta / \delta x^{i}} \frac{\delta}{\delta x^{i}}-\nabla_{\delta / \delta x^{j}} \frac{\delta}{\delta x^{i}}, \frac{\partial}{\partial x^{0}}\right) .
$$

Thus, $\omega_{i j}, i, j \in\{1,2,3\}$, define the vorticity tensor field on $(M, g)$. By using the Jacobi identity

$$
\begin{array}{r}
{[[X, Y], Z]+[[Y, Z], X]+[[Z, X], Y]=0,} \\
\forall X, Y, Z \in \Gamma(\mathrm{TM}),
\end{array}
$$

we deduce that the vorticity tensor field satisfies the identities

$$
\begin{aligned}
\frac{\partial \omega_{i j}}{\partial x^{0}} & =\frac{1}{2}\left\{\frac{\delta a_{i}}{\delta x^{j}}-\frac{\delta a_{j}}{\delta x^{i}}\right\}, \\
\sum_{(i, j, k)}\left\{\frac{\delta \omega_{i j}}{\delta x^{k}}-\omega_{i j} a_{k}\right\} & =0,
\end{aligned}
$$

where $\sum_{(i, j, k)}$ is the cyclic sum with respect to $(i, j, k)$.

Now, we denote by $\bar{g}_{i j}$ the local components of the Riemannian metric $\bar{g}$ induced by $g$ on SM, with respect to the basis $\left\{\delta / \delta x^{i}\right\}$ in $\Gamma(\mathrm{SM})$, and obtain

$$
\bar{g}_{i j}=\bar{g}\left(\frac{\delta}{\delta x^{i}}, \frac{\delta}{\delta x^{j}}\right)=g_{i j}+\Phi^{2} A_{i} A_{j}=g_{i j}+\Phi^{-2} \xi_{i} \xi_{j} .
$$

Then the lone element of $g$ is expressed in terms of threading coframe $\left\{\delta x^{0}, d x^{i}\right\}$ as follows:

$$
d s^{2}=-\Phi^{2}\left(\delta x^{0}\right)^{2}+\bar{g}_{i j} d x^{i} d x^{j}
$$

Also, we define the expansion tensor field $\Theta_{i j}$, the expansion function $\Theta$, and the shear tensor field $\sigma_{i j}$ as follows:

$$
\begin{aligned}
\Theta_{i j} & =\frac{1}{2} \frac{\partial \bar{g}_{i j}}{\partial x^{0}}, \\
\Theta & =\Theta_{i j} \bar{g}^{i j} \\
\sigma_{i j} & =\Theta_{i j}-\frac{1}{3} \Theta \bar{g}_{i j} .
\end{aligned}
$$


Raising and lowering indices $i, j, k, \ldots$, are performed by using $\bar{g}^{i j}$ and $\bar{g}_{i j}$, as in the following examples:

$$
\begin{aligned}
\omega_{j}^{k} & =\bar{g}^{k i} \omega_{i j}, \\
\omega^{k h} & =\bar{g}^{k i} \bar{g}^{h j} \omega_{i j}, \\
\omega_{i j} & =\bar{g}^{i k} \omega_{j}^{k}, \\
\omega_{i j} & =\bar{g}_{i k} \bar{g}_{j h} \omega^{k h} .
\end{aligned}
$$

The expansion and vorticity tensor fields enable us to define the extrinsic curvature tensor field $K$ of the spatial distribution by its local components

$$
K_{j}^{h}=\Theta_{j}^{h}+\Phi^{2} \omega_{j}^{h},
$$

or equivalently by

$$
K_{i j}=\Theta_{i j}+\Phi^{2} \omega_{i j} .
$$

By using (5a) and (15), we see that $K$ is a symmetric tensor field if and only if SM is integrable.

Remark 2. The extrinsic curvature tensor field was intensively used in the $(3+1)$ decomposition of the spacetime (cf. [3, pp $509-516]$ ). As far as we know, the tensor field $K$ given by (14) or (15) is considered here for the first time in a study of the $(1+3)$ threading of spacetime.

Next, in order to justify the tensorial meaning of the above quantities, we define a spatial tensor field $T$ of type $(p, q)$ on $M$, as an $\mathscr{F}(M)$-multilinear mapping:

$$
T: \Gamma\left(\mathrm{SM}^{\star}\right)^{p} \times \Gamma(\mathrm{SM})^{q} \longrightarrow \mathscr{F}(M),
$$

where $\mathrm{SM}^{\star}$ is the dual vector bundle to SM. The local components of $T$, with respect to a threading frame and coframe, are given by

$$
T_{i \cdots}^{k \cdots}=T\left(d x^{k}, \ldots, \frac{\delta}{\delta x^{i}}, \ldots\right)
$$

and satisfy

$$
T_{i \cdots \cdots}^{k \cdots} \frac{\partial \widetilde{x}^{h}}{\partial x^{k}}=\widetilde{T}_{j \cdots}^{h \cdots} \frac{\partial \widetilde{x}^{j}}{\partial x^{i}},
$$

with respect to the coordinate transformations $\widetilde{x}^{a}=$ $\widetilde{x}^{a}\left(x^{0}, x^{i}\right)$ on $M$. For example, $\left\{\omega_{i j}, \bar{g}_{i j}, \theta_{i j}, \sigma_{i j}, K_{i j}\right\}$ and $\left\{a_{i}, c_{i}\right\}$ define spatial tensor fields of types $(0,2)$ and $(0,1)$, respectively.

An important geometric object is the Riemannian spatial connection, which is a metric linear connection $\bar{\nabla}$ on the spatial distribution, given by

$$
\bar{\nabla}_{X} \delta Y=\delta \nabla_{X} \delta Y, \quad \forall X, Y \in \Gamma(\mathrm{TM}),
$$

where $\delta$ is the projection morphism of TM on SM with respect to decomposition (1). Locally, $\bar{\nabla}$ is given by

$$
\bar{\nabla}_{\delta / \delta x^{j}} \frac{\delta}{\delta x^{i}}=\bar{\Gamma}_{i}^{k} \frac{\delta}{\delta x^{k}},
$$

$$
\bar{\nabla}_{\partial / \partial x^{0}} \frac{\delta}{\delta x^{i}}=K_{i}^{k} \frac{\delta}{\delta x^{k}}
$$

where we put

$$
\bar{\Gamma}_{i j}^{k}=\frac{1}{2} \bar{g}^{k h}\left\{\frac{\delta \bar{g}_{h j}}{\delta x^{i}}+\frac{\delta \bar{g}_{h i}}{\delta x^{j}}-\frac{\delta \bar{g}_{i j}}{\delta x^{h}}\right\} .
$$

Throughout the paper, the covariant derivatives defined by $\bar{\nabla}$ will be denoted by a vertical bar "I." As an example, for a spatial tensor field $T=\left(T_{j}^{i}\right)$, we have

$$
\begin{aligned}
T_{\left.i\right|_{k}}^{j} & =\frac{\delta T_{i}^{j}}{\delta x^{k}}+T_{i}^{h} \bar{\Gamma}_{h k}{ }^{j}-T_{h}^{j} \bar{\Gamma}^{h}{ }_{k}, \\
T_{\left.i\right|_{0}}^{j} & =\frac{\partial T_{i}^{j}}{\partial x^{0}}+T_{i}^{h} K_{h}^{j}-T_{h}^{j} K_{i}^{h} .
\end{aligned}
$$

A covariant derivative as in (22a) (resp., (22b)) is called a spatial covariant derivative (resp., temporal covariant derivative) of the spatial tensor field $T$.

Finally, by direct calculations, using the Riemannian spatial connection and the above spatial tensor fields, we express the Levi-Civita connection $\nabla$ on $(M, g)$ as follows:

$$
\begin{aligned}
\nabla_{\delta / \delta x^{j}} \frac{\delta}{\delta x^{i}} & =\bar{\Gamma}_{i}^{k} \frac{\delta}{\delta x^{k}}+\left(\omega_{i j}+\Phi^{-2} \Theta_{i j}\right) \frac{\partial}{\partial x^{0}} \\
& =\bar{\Gamma}_{i}^{k} \frac{\delta}{\delta x^{k}}+\Phi^{-2} K_{i j} \frac{\partial}{\partial x^{0}} \\
\nabla_{\partial / \partial x^{0}} \frac{\delta}{\delta x^{i}} & =\left(\Theta_{i}^{k}+\Phi^{2} \omega_{i}^{k}\right) \frac{\delta}{\delta x^{k}}+b_{i} \frac{\partial}{\partial x^{0}} \\
& =K_{i}^{k} \frac{\delta}{\delta x^{k}}+b_{i} \frac{\partial}{\partial x^{0}} \\
\nabla_{\delta / \delta x^{i}} \frac{\partial}{\partial x^{0}} & =\left(\Theta_{i}^{k}+\Phi^{2} \omega_{i}^{k}\right) \frac{\delta}{\delta x^{k}}+c_{i} \frac{\partial}{\partial x^{0}} \\
& =K_{i}^{k} \frac{\delta}{\delta x^{k}}+c_{i} \frac{\partial}{\partial x^{0}} \\
\nabla_{\partial / \partial x^{0}} \frac{\partial}{\partial x^{0}} & =\Phi^{2} b^{k} \frac{\delta}{\delta x^{k}}+\Psi \frac{\partial}{\partial x^{0}}
\end{aligned}
$$

where we put

$$
b_{i}=a_{i}+c_{i}, \quad i \in\{1,2,3\} .
$$

Remark 3. It is worth mentioning that all the equations we state in the paper are expressed in terms of spatial tensor fields and their covariant derivatives defined by the Riemannian connection.

Remark 4. As the $(1+3)$ threading of spacetime considered in this paper contains as a particular case the $(1+3)$ threading with respect to a unit timelike vector field, we call it the general $(1+3)$ threading of spacetime. The advantage of this general setting on the splitting of spacetime is that it can be applied to any Lorentz metric of a spacetime. 


\section{Bianchi Identities for the Riemannian Spatial Connection}

In earlier literature on the $(1+3)$ threading of spacetime, we find the so-called three-dimensional derivative operator (cf. (4.19) of [4]). With respect to this operator, we have the following remarks:

(i) It is neither a linear connection on $M$ nor a linear connection on SM.

(ii) As a consequence of (i), for the general case when SM is not integrable, then a curvature tensor field for this operator could be not defined.

Contrary to this situation, $\bar{\nabla}$ given by (19a) is a metric linear connection on the vector bundle SM, and therefore it has a curvature tensor field $\bar{R}$ given by

$$
\begin{array}{r}
\bar{R}(X, Y, Z)=\bar{\nabla}_{X} \bar{\nabla}_{Y} \delta Z-\bar{\nabla}_{Y} \bar{\nabla}_{X} \delta Z-\bar{\nabla}_{[X, Y]} \delta Z \\
\forall X, Y, Z \in \Gamma(\mathrm{TM}) .
\end{array}
$$

Locally, we put

$$
\begin{aligned}
& \bar{R}\left(\frac{\delta}{\delta x^{k}}, \frac{\delta}{\delta x^{j}}, \frac{\delta}{\delta x^{i}}\right)=\bar{R}_{i}^{h}{ }_{j k} \frac{\delta}{\delta x^{h}}, \\
& \bar{R}\left(\frac{\delta}{\delta x^{k}}, \frac{\partial}{\partial x^{0}}, \frac{\delta}{\delta x^{i}}\right)=\bar{R}_{i}^{h}{ }_{0 k}^{h} \frac{\delta}{\delta x^{h}},
\end{aligned}
$$

and by using (25), (26a), (26b), (20a), (20b), (5a), and (5b), we obtain

$$
\begin{aligned}
\bar{R}_{i j k}^{h}= & \frac{\delta \bar{\Gamma}_{i j}^{h}}{\delta x^{k}}-\frac{\delta \bar{\Gamma}_{i k}^{h}}{\delta x^{j}}+\bar{\Gamma}_{i j}^{l} \bar{\Gamma}_{l k}^{h}-\bar{\Gamma}_{i k}^{l} \bar{\Gamma}_{l j}^{h} \\
& -2 K_{i}^{h} \omega_{j k}, \\
\bar{R}_{i 0 k}^{h}= & K_{i \mid k}^{h}-\frac{\partial \bar{\Gamma}_{i k}^{h}}{\partial x^{0}}+K_{i}^{h} a_{k} .
\end{aligned}
$$

Since $\bar{\nabla}$ is a metric linear connection, we have

$$
\bar{R}_{i l 0 k}+\bar{R}_{l i 0 k}=0
$$

where we put

$$
\bar{R}_{i l 0 k}=\bar{g}_{l h} \bar{R}_{i \quad k}^{h}
$$

As a consequence of (28) we deduce that $\bar{R}_{i 0 k}^{i}=0$, which implies

$$
\Theta_{\mid k}=\frac{\partial \Theta}{\partial x^{k}}-A_{k} \frac{\partial \Theta}{\partial x^{0}}=\frac{\bar{\Gamma}_{i k}^{i}}{\partial x^{0}}-\Theta a_{k}
$$

via (27b) and (14). Thus, in any cosmological model of a $4 D$ universe, the expansion function must satisfy the system of $P D E$ given by (30).
Remark 5. Note that $\bar{R}_{i j k}^{h}$ and $\bar{R}_{i \quad k \text { define spatial tensor }}^{h}$ fields of types $(1,3)$ and $(1,2)$, respectively. Also, from (27b) we see that $\partial \bar{\Gamma}_{i k}^{h} / \partial x^{0}$ define a spatial tensor field of type $(1,2)$. However, $\bar{\Gamma}_{i k}^{h}$ do not define a spatial tensor field.

Remark 6. Comparing (27a) with (15.4) from [5], we see that the so-called Zelmanov curvature tensor field is given by the first four terms from (27a). Moreover, from (15.5) of [5] we see that such a tensor field becomes a curvature tensor field, if and only if SM is an integrable distribution.

Next, we extend the Riemannian spatial connection $\bar{\nabla}$ on SM to a linear connection $\widetilde{\nabla}$ on $M$ given by

$$
\widetilde{\nabla}_{X} Y=\bar{\nabla}_{X} \mathcal{S}+\mathscr{T} \nabla_{X} \mathscr{T} Y, \quad \forall X, Y \in \Gamma(\mathrm{TM}),
$$

where $\mathscr{T}$ is the projection morphism of TM on VM with respect to (1). Clearly, $\widetilde{\nabla}$ coincides with $\bar{\nabla}$ on SM and therefore locally is given by (20a), (20b), and

$$
\begin{aligned}
& \widetilde{\nabla}_{\delta / \delta x^{i}} \frac{\partial}{\partial x^{0}}=c_{i} \frac{\partial}{\partial x^{0}}, \\
& \widetilde{\nabla}_{\delta / \delta x^{0}} \frac{\partial}{\partial x^{0}}=\Psi \frac{\partial}{\partial x^{0}} .
\end{aligned}
$$

We recall that the torsion and curvature tensor fields of $\widetilde{\nabla}$ are given by

$$
\begin{aligned}
\widetilde{T}(X, Y) & =\widetilde{\nabla}_{X} Y-\widetilde{\nabla}_{Y} X-[X, Y], \\
\widetilde{R}(X, Y, Z) & =\widetilde{\nabla}_{X} \widetilde{\nabla}_{Y} Z-\widetilde{\nabla}_{Y} \widetilde{\nabla}_{X} Z-\widetilde{\nabla}_{[X, Y]} Z .
\end{aligned}
$$

Then, by direct calculations, using (33a), (33b), (20a), (20b), (32a), (32b), (5a), (5b), (23c), (26a), and (26b), we deduce that

$$
\begin{aligned}
& \widetilde{T}\left(\frac{\delta}{\delta x^{j}}, \frac{\delta}{\delta x^{i}}\right)=-2 \omega_{i j} \frac{\partial}{\partial x^{0}}, \\
& \widetilde{T}\left(\frac{\partial}{\partial x^{0}}, \frac{\delta}{\delta x^{i}}\right)=K_{i}^{j} \frac{\delta}{\delta x^{j}}-b_{i} \frac{\partial}{\partial x^{0}},
\end{aligned}
$$

$$
\begin{aligned}
& \widetilde{R}\left(\frac{\delta}{\delta x^{h}}, \frac{\delta}{\delta x^{k}}, \frac{\delta}{\delta x^{i}}\right)=\bar{R}_{i k h}^{j} \frac{\delta}{\delta x^{j}}, \\
& \widetilde{R}\left(\frac{\delta}{\delta x^{k}}, \frac{\partial}{\partial x^{0}}, \frac{\delta}{\delta x^{i}}\right)=\bar{R}_{i 0 k}^{j} \frac{\delta}{\delta x^{j}} .
\end{aligned}
$$

Now, in order to find some Bianchi identities for the Riemannian spatial connection, we recall that the Bianchi identities for the linear connection $\widetilde{\nabla}$ are given by (cf. [6, p. 135])

$$
\begin{aligned}
& \sum_{(X, Y, Z)}\left\{\left(\widetilde{\nabla}_{X} \widetilde{T}\right)(Y, Z)+\widetilde{T}(\widetilde{T}(X, Y), Z)\right. \\
& \quad-\widetilde{R}(X, Y, Z)\}=0, \\
& \sum_{(X, Y, Z)}\left\{\left(\widetilde{\nabla}_{X} \widetilde{R}\right)(Y, Z, U)+\widetilde{R}(\widetilde{T}(X, Y), Z, U)\right\}=0,
\end{aligned}
$$


where $\sum_{(X, Y, Z)}$ is the cyclic sum with respect to $(X, Y, Z)$. In order to use (35a), we note that

$$
\begin{aligned}
& \widetilde{T}\left(\widetilde{T}\left(\frac{\delta}{\delta x^{k}}, \frac{\delta}{\delta x^{j}}\right), \frac{\delta}{\delta x^{i}}\right) \\
& =-2 K_{i}^{h} \omega_{j k} \frac{\delta}{\delta x^{h}}+2 b_{i} \omega_{j k} \frac{\partial}{\partial x^{0}}, \\
& \left(\widetilde{\nabla}_{\delta / \delta x^{k}} \widetilde{T}\right)\left(\frac{\delta}{\delta x^{j}}, \frac{\delta}{\delta x^{i}}\right)=-2\left(\omega_{i j \mid k}+\omega_{i j} c_{k}\right) \frac{\partial}{\partial x^{0}} .
\end{aligned}
$$

Then, take $X=\delta / \delta x^{k}, Y=\delta / \delta x^{j}$, and $Z=\delta / \delta x^{i}$ in (35a) and, by using (36a), (36b), and (34a), we infer that the spatial component in (35a) is expressed as follows:

$$
\sum_{(i, j, k)}\left\{\bar{R}_{i j k}^{h}+2 K_{i}^{h} \omega_{j k}\right\}=0
$$

Taking temporal part in (35a) and any other triplet $(X, Y, Z)$ from the threading frame we obtain the identities from (9a) and (9b).

Next, take $X=\delta / \delta x^{k}, Y=\delta / \delta x^{j}, Z=\delta / \delta x^{i}$, and $U=$ $\delta / \delta x^{h}$ in (35b), and, by using (31), (34a), (34c) and (34d), we obtain

$$
\sum_{(i, j, k)}\left\{\bar{R}_{h i j \mid k}^{l}+\bar{R}_{h 0 i \mid k}^{l} \omega_{j k}\right\}=0
$$

Finally, take $X=\partial / \partial x^{0}, Y=\delta / \delta x^{j}, Z=\delta / \delta x^{i}$, and $U=$ $\delta / \delta x^{h}$ in (35b), and, by using (31), (34a), (34b), (34c), (34d), and $(23 \mathrm{c})$, we deduce the identity

$$
\begin{gathered}
\bar{R}_{h i j \mid 0}^{l}+\bar{R}_{h 0 i \mid j}^{l}-\bar{R}_{h 0 j \mid i}^{l}+\bar{R}_{h i k}^{l} K_{j}^{k}-\bar{R}_{h j k}^{l} K_{i}^{k} \\
+a_{j} \bar{R}_{h 0 i}^{l}-a_{i} \bar{R}_{h 0 j}^{l}=0 .
\end{gathered}
$$

The other identities obtained from (35b) either are trivial or do not involve the curvature tensor $\bar{R}$ of $\bar{\nabla}$. Thus, we are entitled to call (37), (38), and (39) the Bianchi identities for the Riemannian spatial connection.

We close the section with some comments on these identities. As far as we know, the above Bianchi identities are stated here for the first time in the literature. They represent a generalization of usual Bianchi identities on a 3-dimensional Riemannian manifold. Indeed, if the spatial distribution is integrable, that is, the vorticity tensor field vanishes identically on $M$, then (37) and (38) become

$$
\begin{gathered}
\sum_{(i, j, k)}\left\{\bar{R}_{i j k}^{l}\right\}=0, \\
\sum_{(i, j, k)}\left\{\bar{R}_{h i j \mid k}^{l}\right\}=0,
\end{gathered}
$$

which are the well-known Bianchi identities on the 3-dimensional leaves of SM. Moreover, in this case, by using (14) and (27a) and (27b), we deduce that

$$
\begin{aligned}
K_{i}^{j} & =\Theta_{i}^{j}, \\
\bar{R}_{i 0 h}^{j} & =\Theta_{i \mid h}^{j}-\frac{\partial \bar{\Gamma}_{i h}^{j}}{\partial x^{0}}+\Theta_{i}^{j} a_{h}, \\
\bar{R}_{i k h}^{j} & =\frac{\delta \bar{\Gamma}_{i k}^{j}}{\delta x^{h}}-\frac{\delta \bar{\Gamma}_{i h}^{j}}{\delta x^{k}}+\bar{\Gamma}_{i k}^{l} \bar{\Gamma}_{l h}^{j}-\bar{\Gamma}_{i h}^{l} \bar{\Gamma}_{l k}^{j} .
\end{aligned}
$$

Finally, identity (39) becomes

$$
\begin{gathered}
\bar{R}_{h i j \mid 0}^{l}+\bar{R}_{h 0 i \mid j}^{l}-\bar{R}_{h 0 j \mid i}^{l}+\bar{R}_{h i k}^{l} \Theta_{j}^{k}-\bar{R}_{h j k}^{l} \Theta_{i}^{k} \\
+a_{j} \bar{R}_{h 0 i}^{l}-a_{i} \bar{R}_{h 0 j}^{l}=0 .
\end{gathered}
$$

\section{Structure Equations Induced by the $(1+3)$ Threading of Spacetime}

Let $(M, g)$ be a $4 D$ spacetime and $\nabla$ be the Levi-Civita connection defined by the Lorentz metric $g$. Denote by $R$ the curvature tensor field of $\nabla$ given by (33b) wherein we remove the tilde. Then, consider the following local components of $R$ with respect to the threading frame $\left\{\partial / \partial x^{0}, \delta / \delta x^{i}\right\}$ :

$$
\begin{aligned}
& R\left(\frac{\delta}{\delta x^{k}}, \frac{\delta}{\delta x^{j}}, \frac{\delta}{\delta x^{i}}\right)=R_{i}^{h}{ }_{j k} \frac{\delta}{\delta x^{h}}+R_{i}{ }^{0}{ }_{j k} \frac{\partial}{\partial x^{0}} \\
& R\left(\frac{\delta}{\delta x^{k}}, \frac{\partial}{\partial x^{0}}, \frac{\delta}{\delta x^{i}}\right)=R_{i}^{h}{ }_{0 k} \frac{\delta}{\delta x^{h}}+R_{i}^{0}{ }_{0 k} \frac{\partial}{\partial x^{0}} .
\end{aligned}
$$

Now, comparing (A.4) and (A.8) from Appendix A with (43a) and $(43 \mathrm{~b})$, respectively, we obtain

$$
\begin{aligned}
& R_{i j k}^{h}=\bar{R}_{i j k}^{h}+\Phi^{-2}\left(K_{i j} K_{k}^{h}-K_{i k} K_{j}^{h}\right), \\
& R_{i j k}^{0}=\Phi^{-2}\left(K_{i j \mid k}-K_{i k \mid j}+K_{i k} c_{j}-K_{i j} c_{k}\right)-2 b_{i} \omega_{j k}, \\
& R_{i 0 k}^{h}=\bar{R}_{i 0 k}^{h}+b_{i} K_{k}^{h}-b^{h} K_{i k}, \\
& R_{i 0 k}^{0}=b_{i \mid k}+b_{i} b_{k}-\Phi^{-2}\left(K_{i k \mid 0}+K_{i j} K_{k}^{j}-\Psi K_{i k}\right) .
\end{aligned}
$$

By using (15), (6a), (6b), (6c), and (6d), we deduce that

$$
\begin{aligned}
& K_{i j \mid k}=\Theta_{i j \mid k}+\Phi^{2}\left(\omega_{i j \mid k}+2 \omega_{i j} c_{k}\right), \\
& K_{i j \mid 0}=\Theta_{i j \mid 0}+\Phi^{2}\left(\omega_{i j \mid 0}+2 \Psi \omega_{i j}\right) .
\end{aligned}
$$


Taking account of (45a), (45b), (14), and (15) into (44a), (44b), $(44 \mathrm{c})$, and $(44 \mathrm{~d})$, we infer that

$$
\begin{aligned}
R_{i j k}^{h}= & \bar{R}_{i j k}^{h}+\left(\omega_{i j}+\Phi^{-2} \Theta_{i j}\right)\left(\Theta_{k}^{h}+\Phi^{2} \omega_{k}^{h}\right) \\
& -\left(\omega_{i k}+\Phi^{-2} \Theta_{i k}\right)\left(\Theta_{j}^{h}+\Phi^{2} \omega_{j}^{h}\right), \\
R_{i j k}{ }^{h}= & \Phi^{-2}\left(\Theta_{i j \mid k}-\Theta_{i k \mid j}+\Theta_{i k} c_{j}-\Theta_{i j} c_{k}\right)+\omega_{i j \mid k} \\
& -\omega_{i k \mid j}+\omega_{i j} c_{k}-\omega_{i k} c_{j}-2 b_{i} \omega_{j k}, \\
R_{i 0 k}^{h}= & \bar{R}_{i 0 k}^{h}+b_{i} \Theta_{k}^{h}-b^{h} \Theta_{i k}+\Phi^{2}\left(b_{i} \omega_{k}^{h}-b^{h} \omega_{i k}\right), \\
R_{i 0 k}^{0}= & b_{i \mid k}+b_{i} b_{k}-\Phi^{-2}\left(\Theta_{i k \mid 0}-\Psi \Theta_{i k}\right)-\omega_{i k \mid 0} \\
& -\Psi \omega_{i k}-\left(\omega_{i j}+\Phi^{-2} \Theta_{i j}\right)\left(\Theta_{k}^{j}+\Phi^{2} \omega_{k}^{j}\right) .
\end{aligned}
$$

Next, by using the local components of the curvature tensor fields $R$ and $\bar{R}$ of type (0,4) (see (A.9) and (A.10)), from (44a), (44b), (44c), (44d), (46a), (46b), (46c), and (46d) we obtain

$$
\begin{aligned}
R_{i l j k} & =\bar{R}_{i l j k}+\Phi^{-2}\left(K_{i j} K_{l k}-K_{i k} K_{l j}\right), \\
R_{i 0 j k} & =K_{i k \mid j}-K_{i j \mid k}+K_{i j} c_{k}-K_{i k} c_{j}+2 \Phi^{2} b_{i} \omega_{j k}, \\
R_{i l 0 k} & =\bar{R}_{i l 0 k}+b_{i} K_{l k}-b_{l} K_{i k}, \\
R_{i 00 k} & =K_{i k \mid 0}+K_{i j} K_{k}^{j}-\Psi K_{i k}-\Phi^{-2}\left(b_{i \mid k}+b_{i} b_{k}\right), \\
R_{i l j k} & =\bar{R}_{i j k}+\Phi^{2}\left\{\left(\omega_{i j}+\Phi^{-2} \Theta_{i j}\right)\left(\omega_{l k}+\Phi^{-2} \Theta_{l k}\right)\right. \\
- & \left.\left(\omega_{i k}+\Phi^{-2} \Theta_{i k}\right)\left(\omega_{l j}+\Phi^{-2} \Theta_{l j}\right)\right\}, \\
R_{i 0 j k} & =\Theta_{i k \mid j}-\Theta_{i j \mid k}+\Theta_{i j} c_{k}-\Theta_{i k} c_{j}+\Phi^{2}\left\{\omega_{i k \mid j}\right. \\
- & \left.\omega_{i j \mid k}+\omega_{i k} c_{j}-\omega_{i j} c_{k}+2 b_{i} \omega_{j k}\right\}, \\
R_{i l 0 k} & =\bar{R}_{i l 0 k}+b_{i} \Theta_{l k}-b_{l} \Theta_{i k}+\Phi^{2}\left(b_{i} \omega_{l k}-b_{l} \omega_{i k}\right), \\
R_{i 00 k} & =\Theta_{i k \mid 0}-\Psi \Theta_{i k}+\Phi^{2}\left\{\omega_{i k \mid 0}+\Psi \omega_{i k}\right. \\
+ & \left.\left(\omega_{i j}+\Phi^{-2} \Theta_{i j}\right)\left(\Theta_{k}^{j}+\Phi^{2} \omega_{k}^{j}\right)-b_{i \mid k}-b_{i} b_{k}\right\} .
\end{aligned}
$$

With the theory of hypersurfaces of the spacetime in mind, we call (47a) and (48a) \{resp., (47b), (48b), and (48c)\} the Gauss equations (resp., Codazzi equations) for the spatial distribution SM in the ambient space $(M, g)$. Also, all the equations from (47a), (47b), (47c), (47d), (48a), (48b), (48c), and $(48 \mathrm{~d})$ will be called structure equations induced by the $(1+3)$ threading formalism. They have an important role in the next sections.

Now, taking into account the symmetries of $R$, we deduce some identities for $\bar{R}$ and for kinematic quantities. First, using well-known identities for $R$ and taking into account (47a) and (48a), we obtain the following identities for $\bar{R}$ :

$$
\begin{aligned}
& \bar{R}_{i l j k}+\bar{R}_{i l k j}=0, \\
& \bar{R}_{i l j k}+\bar{R}_{l i j k}=0, \\
& \bar{R}_{i l j k}-\bar{R}_{j k i l} \\
& =\Phi^{-2}\left\{K_{i k} K_{l j}+K_{j i} K_{k l}-K_{i j} K_{l k}-K_{j l} K_{k i}\right\} \\
& =2\left\{\Theta_{i k} \omega_{l j}+\Theta_{l j} \omega_{i k}+\Theta_{i j} \omega_{k l}+\Theta_{k l} \omega_{j i}\right\} .
\end{aligned}
$$

Also, taking into account that

$$
R_{i 0 j k}=-R_{j k 0 i}
$$

and by using (47b), (47c), (48b), and (48c), we deduce that

$$
\begin{aligned}
\bar{R}_{j k 0 i}= & K_{i j \mid k}-K_{i k \mid j}+K_{i k} c_{j}-K_{i j} c_{k}-2 \Phi^{2} b_{i} \omega_{j k} \\
& -b_{j} K_{k i}+b_{k} K_{j i} \\
= & \Theta_{i j \mid k}-\Theta_{i k \mid j}+\Theta_{i j} a_{k}-\Theta_{i k} a_{j} \\
& +\Phi^{2}\left\{\omega_{i j \mid k}-\omega_{i k \mid j}+\omega_{i k} a_{j}-\omega_{i j} a_{k}-2 b_{i} \omega_{j k}\right\} .
\end{aligned}
$$

Finally, using the identity

$$
R_{i 00 k}=R_{k 00 i}
$$

and taking the symmetric and skew-symmetric parts in (47d) and (48d), we infer that

$$
\begin{aligned}
& R_{i 00 k}=\frac{1}{2}\left\{K_{i k \mid 0}+K_{k i \mid 0}+K_{i j} K_{k}^{j}+K_{k j} K_{i}^{j}\right. \\
& \left.-\Psi\left(K_{i k}+K_{i k}\right)-\Phi^{-2}\left(b_{i \mid k}+b_{k \mid i}\right)\right\}-\Phi^{2} b_{i} b_{k}, \\
& R_{i 00 k}=\Theta_{i k \mid 0}-\Psi \Theta_{i k}+\Theta_{i j} \theta_{k}^{j}+\Phi^{4} \omega_{i j} \omega_{k}^{j}-\Phi^{2} b_{i} b_{k} \\
& \quad-\frac{1}{2} \Phi^{2}\left(b_{i \mid k}+b_{k \mid i}\right), \\
& K_{i k \mid 0}-K_{k i \mid 0}+K_{i j} K_{k}^{j}-K_{k j} K_{i}^{j}-\Psi\left(K_{i k}-K_{k i}\right) \\
& -\Phi^{2}\left(b_{i \mid k}-b_{k \mid i}\right)=0, \\
& \omega_{i k \mid 0}=-\Psi \omega_{i k}+\Phi^{2}\left\{\omega_{k j} \Theta_{i}^{j}-\omega_{i j} \Theta_{k}^{j}\right. \\
& \left.+\frac{1}{2}\left(b_{i \mid k}-b_{k \mid i}\right)\right\} .
\end{aligned}
$$

In particular, suppose that $(M, g)$ is a vorticity-free spacetime; that is, the vorticity tensor field vanishes identically on $M$. Then, from (49c) and (51) we deduce that the curvature tensor field of the Riemannian spatial connection satisfies identities (49a) and (49b) and the following:

$$
\begin{aligned}
\bar{R}_{i l j k} & =\bar{R}_{j k i l}, \\
\bar{R}_{j k o i} & =\Theta_{i j \mid k}-\Theta_{i k \mid j}+\Theta_{i j} a_{k}-\Theta_{i k} a_{j} .
\end{aligned}
$$


Also, from (54b) we see that

$$
b_{i \mid k}=b_{k \mid i}
$$

and (48a), (48b), (48c), and (48d) become

$$
\begin{aligned}
R_{i l j k} & =\bar{R}_{i l j k}+\Phi^{-2}\left(\Theta_{i j} \Theta_{l k}-\Theta_{i k} \Theta_{l j}\right), \\
R_{i 0 j k} & =\Theta_{i k \mid j}-\Theta_{i j \mid k}+\Theta_{i j} c_{k}-\Theta_{i k} c_{j}, \\
R_{i l 0 k} & =\bar{R}_{i l 0 k}+\Theta_{l k} b_{i}-\Theta_{i k} b_{l}, \\
R_{i 00 k} & =\Theta_{i k \mid 0}-\Psi \Theta_{i k}+\Theta_{i j} \Theta_{k}^{j}-\Phi^{2}\left(b_{i \mid k}+b_{i} b_{k}\right) .
\end{aligned}
$$

\section{Ricci Tensor Field and Scalar Curvature of a Spacetime Expressed in terms of Spatial Tensor Fields}

Let $(M, g)$ be a $4 D$ spacetime and $\left\{E_{k}\right\}$ be an orthonormal basis in $\Gamma(\mathrm{SM})$. Then, $\left\{\Phi^{-1}\left(\partial / \partial x^{0}\right), E_{k}\right\}$ is an orthonormal frame field on $M$. According to [7, p. 87], the Ricci tensor of $(M, g)$ is given by

$$
\begin{aligned}
\operatorname{Ric}(X, Y)= & \sum_{k=1}^{3} R\left(E_{k}, X, E_{k}, Y\right) \\
& -\Phi^{-2} R\left(\frac{\partial}{\partial x^{0}}, X, \frac{\partial}{\partial x^{0}}, Y\right),
\end{aligned}
$$

for all $X, Y \in \Gamma(\mathrm{TM})$. Now, we express $E_{k}$ as follows:

$$
E_{k}=E_{k}^{i} \frac{\delta}{\delta x^{i}}
$$

and we obtain

$$
\bar{g}^{i j}=\sum_{k=1}^{3} E_{k}^{i} E_{k}^{j} .
$$

Then we consider the following local components of Ric with respect to the threading frame $\left\{\partial / \partial x^{0}, \delta / \delta x^{i}\right\}$ :

$$
\begin{aligned}
& R_{i j}=\operatorname{Ric}\left(\frac{\delta}{\delta x^{j}}, \frac{\delta}{\delta x^{i}}\right), \\
& R_{i 0}=\operatorname{Ric}\left(\frac{\partial}{\partial x^{0}}, \frac{\delta}{\delta x^{i}}\right), \\
& R_{00}=\operatorname{Ric}\left(\frac{\partial}{\partial x^{0}}, \frac{\partial}{\partial x^{0}}\right),
\end{aligned}
$$

and, by using (59), (60), (61a), (61b), (61c), and (A.9) into (58), we obtain

$$
\begin{aligned}
& R_{i j}=\bar{g}^{l k} R_{i l j k}+\Phi^{-2} R_{i 00 j}, \\
& R_{i 0}=\bar{g}^{l k} R_{i l 0 k}=-\bar{g}^{l k} R_{k 0 i l}, \\
& R_{00}=-\bar{g}^{i k} R_{i 00 k} .
\end{aligned}
$$

Now, by using (47a), (47b), (47c), (47d), (48a), (48b), (48c), (48d), (53a), and (53b) in (62a), (62b), and (62c), we deduce that

$$
\begin{aligned}
R_{i j}= & \bar{R}_{i j k}^{k}+\Phi^{-2}\left\{(\Theta-\Psi) K_{i j}+K_{i j \mid 0}\right\}-b_{i \mid j}-b_{i} b_{j}, \\
R_{i 0}= & \bar{R}_{i 0 k}^{k}+\Theta b_{i}-K_{i k} b^{k} \\
= & K_{i \mid k}^{k}-\Theta_{\mid i}+\Theta c_{i}-c_{k} K_{I}^{k}+2 \Phi^{2} \omega_{i k} b^{k}, \\
R_{00}= & \Psi \Theta-\Theta_{\mid 0}-K_{h}^{k} K_{k}^{h}+\Phi^{2}\left\{b^{2}+b_{\mid k}^{k}\right\}, \\
R_{i j}= & \bar{R}_{i j k}^{k}+\Phi^{-2}\left\{(\Theta-\Psi) \Theta_{i j}+\Theta_{i j \mid 0}\right\}+\omega_{i j \mid 0} \\
& +(\Theta+\Psi) \omega_{i j}-b_{i \mid j}-b_{i} b_{j}, \\
R_{i 0}= & \bar{R}_{i}^{k}+{ }^{k}+b_{i}-\Theta_{i k} b^{k}-\Phi^{2} \omega_{i k} b^{k}, \\
= & \Theta_{i \mid k}^{k}-\Theta_{\mid i}+\Theta c_{i}-\Theta_{i k} c^{k} \\
& -\Phi^{2}\left(\omega_{i \mid k}^{k}+c_{k} \omega_{i}^{k}-\omega_{i k} b^{k}\right), \\
& +\Phi^{2}\left(b^{2}+b_{\mid k}^{k}\right), \\
R_{00}= & \Psi \Theta-\Theta_{\mid 0}-\sigma^{2}-\frac{1}{3} \Theta^{2}+\Phi^{4} \omega^{2} \\
&
\end{aligned}
$$

where we put

$$
\begin{aligned}
& \sigma^{2}=\sigma_{h k} \sigma^{h k}, \\
& \omega^{2}=\omega_{h k} \omega^{h k}, \\
& b^{2}=b_{k} b^{k} .
\end{aligned}
$$

Next, we take the symmetric and skew-symmetric parts in (63a) and (64a) and obtain

$$
\begin{aligned}
R_{i j}= & \bar{R}_{i j}+\Phi^{-2}\left\{(\Theta-\Psi) \Theta_{i j}+\Theta_{i j \mid 0}\right\} \\
& -\frac{1}{2}\left(b_{i \mid j}+b_{j \mid i}\right)-b_{i} b_{j}, \\
\frac{1}{2}\left(\bar{R}_{i j k}^{k}-\bar{R}_{j i k}^{k}\right)= & \frac{1}{2}\left(b_{i \mid j}-b_{j \mid i}\right)-\omega_{i j \mid 0} \\
& -(\Theta+\Psi) \omega_{i j},
\end{aligned}
$$

where we put

$$
\bar{R}_{i j}=\frac{1}{2}\left(\bar{R}_{i j k}^{k}+\bar{R}_{j i k}^{k}\right) .
$$

The spatial with local components $\bar{R}_{i j}$ are called the spatial Ricci tensor of the spacetime $(M, g)$.

The above formulas for Ricci tensor enable us to obtain a new formula for the scalar curvature $\mathbf{R}$ of $(M, g)$ in terms of spatial tensor fields. We start with $\mathbf{R}$ given by

$$
\begin{aligned}
\mathbf{R} & =\sum_{k=1}^{3} \operatorname{Ric}\left(E_{k}, E_{k}\right)-\Phi^{-2} \operatorname{Ric}\left(\frac{\partial}{\partial x^{0}}, \frac{\partial}{\partial x^{0}}\right) \\
& =\bar{g}^{i j} R_{i j}-\Phi^{-2} R_{00} .
\end{aligned}
$$


Then by direct calculations, using (66a) and (64c) into (68), we obtain

$$
\begin{aligned}
\mathbf{R}= & \overline{\mathbf{R}}+\Phi^{-2}\left\{\frac{4}{3} \Theta^{2}-2 \Psi \Theta+2 \Theta_{\mid 0}\right\}-\Phi^{2} \omega^{2}-2 b^{2} \\
& -2 b_{\mid k}^{k},
\end{aligned}
$$

where we put

$$
\overline{\mathbf{R}}=\bar{g}^{i j} \bar{R}_{i j}
$$

We call $\overline{\mathbf{R}}$ the spatial scalar curvature of the spacetime $(M, g)$. Note that both $\bar{R}_{i j}$ and $\overline{\mathbf{R}}$ are related to the geometry of the spatial distribution SM which is not necessarily supposed to be integrable.

\section{The Splitting of Both the Einstein Gravitational Tensor Field and the Energy- Momentum Tensor Field}

We start with the Einstein gravitational tensor field $G$ of $(M, g)$ given by

$$
G(X, Y)=\operatorname{Ric}(X, Y)-\frac{\mathbf{R}}{2} g(X, Y),
$$

$$
\forall X, Y \in \Gamma(\mathrm{TM}) .
$$

Then, with respect to the threading frame field $\left\{\partial / \partial x^{0}, \delta / \delta x^{i}\right\}$, we have

$$
\begin{aligned}
G_{i j} & =G\left(\frac{\delta}{\delta x^{j}}, \frac{\delta}{\delta x^{i}}\right)=R_{i j}-\frac{\mathbf{R}}{2} g_{i j}, \\
G_{i 0} & =G\left(\frac{\partial}{\partial x^{0}}, \frac{\delta}{\delta x^{i}}\right)=R_{i 0}, \\
G_{00} & =G\left(\frac{\partial}{\partial x^{0}}, \frac{\partial}{\partial x^{0}}\right)=R_{00}+\Phi^{2} \frac{\mathbf{R}}{2} .
\end{aligned}
$$

By using (66a), (64b), (64c), and (69) into (72a), (72b), and (72c), we obtain

$$
\begin{aligned}
G_{i j} & =\bar{G}_{i j}+\left(b^{2}+b_{\mid k}^{k}+\frac{1}{2} \Phi^{2} \omega^{2}\right) \bar{g}_{i j}-\frac{1}{2}\left(b_{i \mid j}+b_{j \mid i}\right) \\
& -b_{i} b_{j}+\Phi^{-2}\left\{(\Theta-\Psi) \Theta_{i j}+\Theta_{i j \mid 0}\right. \\
& \left.-\left(\Theta_{\mid 0}-\Psi \Theta+\frac{2}{3} \Theta^{2}+\frac{1}{2} \sigma^{2}\right) \bar{g}_{i j}\right\}, \\
G_{i 0} & =\bar{R}_{i 0 k}^{k}-\bar{R}_{j}^{k}+\Theta b_{i}-\Theta_{i k} b^{k}-\Phi^{2} \omega_{i k}=\Theta_{i \mid k}^{k} \\
& -\Theta_{\mid i}+\Theta c_{i}-\Theta_{i k} c^{k}-\Phi^{2}\left(\omega_{i \mid k}^{k}+c_{k} \omega_{i}^{k}-2 \omega_{i k} b^{k}\right), \\
G_{00} & =\frac{1}{2}\left(\Phi^{2} \overline{\mathbf{R}}+\frac{2}{3} \Theta^{2}-\sigma^{2}+\Phi^{4} \omega^{2}\right),
\end{aligned}
$$

where we put

$$
\bar{G}_{i j}=\bar{R}_{i j}-\frac{\overline{\mathbf{R}}}{2} \bar{g}_{i j}
$$

The spatial tensor field $\bar{G}$ with local components $\bar{G}_{i j}$ is called the $3 D$ Einstein gravitational tensor field of the spacetime $(M, g)$.

Now, in order to give a coordinate-free formula for the energy-momentum tensor field, we consider a spatial 1-form $\omega$ and a spatial tensor field $S$ of type $(0,2)$. Then, we define a 1 -form and a tensor field of type $(0,2)$ on $M$, denoted by the same symbols and given by

$$
\begin{gathered}
\omega(X)=\omega(\mathcal{S} X), \\
S(X, Y)=S(\mathcal{S} X, \mathcal{S} Y),
\end{gathered}
$$

for all $X, Y \in \Gamma(\mathrm{TM})$. As an example, the Riemannian metric $\bar{g}$ on SM defines a symmetric tensor field $\bar{g}$ on $M$ given by

$$
\bar{g}(X, Y)=\bar{g}(\mathcal{S} X, \mathcal{S} Y), \quad \forall X, Y \in \Gamma(\mathrm{TM}) .
$$

Note that $\bar{g}$ from (76) coincides with the tensor field $h$ given by its local components in formula (4.10) of [4]. Also, we need the 1 -form $u$ induced by the unit vector field $U=\Phi^{-1} \partial / \partial x^{0}$ by the formula

$$
u(X)=\Phi^{-1} g\left(X, \frac{\partial}{\partial x^{0}}\right), \quad \forall X \in \Gamma(\mathrm{TM}) .
$$

Based on these geometric objects, we claim that the energymomentum tensor field $T$ measured by an observer moving with the unit 4-velocity $U$ has the following coordinate-free expression:

$$
\begin{aligned}
T(X, Y)= & \rho u(X) u(Y)+q(X) u(Y)+q(Y) u(X) \\
& +p \bar{g}(X, Y)+\pi(X, Y)
\end{aligned}
$$

$\forall X, Y \in \Gamma(\mathrm{TM})$.

Here, $\rho$ and $p$ are the relativistic energy density and the relativistic pressure, respectively, while $q$ is a 1-form on $M$ defined by a spatial 1-form as in (75a) and $\pi$ is a symmetric and trace-free tensor field on $M$ defined by a spatial tensor field as in (75b). Now, take $X=Y=U$ in (78) and obtain

$$
\rho=T(U, U) .
$$

Then we put

$$
T_{00}=T\left(\frac{\partial}{\partial x^{0}}, \frac{\partial}{\partial x^{0}}\right),
$$

and (79) becomes

$$
T_{00}=\Phi^{2} \rho
$$

Similarly, take $X=U$ and $Y=S Y$ in (78) and deduce that

$$
q(\delta Y)=-T(U, \delta Y)
$$

Now, we put

$$
\begin{aligned}
T_{i 0} & =T\left(\frac{\partial}{\partial x^{0}}, \frac{\delta}{\delta x^{i}}\right), \\
q_{i} & =q\left(\frac{\delta}{\delta x^{i}}\right),
\end{aligned}
$$


and, taking $Y=\delta / \delta x^{i}$ in (82), we infer that

$$
T_{i 0}=-\Phi q_{i} \text {. }
$$

Finally, denote

$$
\begin{aligned}
T_{i j} & =T\left(\frac{\delta}{\delta x^{j}}, \frac{\delta}{\delta x^{i}}\right), \\
\pi_{i j} & =\pi\left(\frac{\delta}{\delta x^{j}}, \frac{\delta}{\delta x^{i}}\right),
\end{aligned}
$$

and, taking $X=\delta / \delta x^{j}$ and $Y=\delta / \delta x^{i}$ in (78), we obtain

$$
T_{i j}=p \bar{g}_{i j}+\pi_{i j}
$$

Contracting (86) by $\bar{g}^{i j}$ and taking into account that $\pi$ is defined by a trace-free spatial tensor field, we infer that

$$
\begin{aligned}
p & =\frac{1}{3} \bar{g}^{i j} T_{i j}, \\
\pi_{i j} & =T_{i j}-\frac{1}{3}\left(T_{h k} \bar{g}^{h k}\right) \bar{g}_{i j} .
\end{aligned}
$$

Now, taking into account (79)-(87b), it is clear that (78) represents the coordinate-free version of (5.9) from [4, p. 91]. Thus $q$ and $\pi$ from (78) are the relativistic momentum density and the relativistic anisotropic (trace-free) stress tensor field, respectively.

\section{A New Splitting of Einstein Field Equations with respect to General $(1+3)$ Threading of Spacetime}

We start this section with the coordinate-free form of Einstein field equations (EFE) expressed as follows:

$$
G(X, Y)+\Lambda g(X, Y)=8 \pi \mathbf{G T}(X, Y),
$$

$\forall X, Y \in \Gamma(\mathrm{TM})$,

where $\Lambda$ is the cosmological constant and $\mathbf{G}$ is the Newton constant. Now, take $X=\delta / \delta x^{j}$ and $Y=\delta / \delta x^{i}$ in (88) and, by using (73a), (10), and (86), we obtain

$$
\begin{aligned}
\bar{G}_{i j} & +\left(b^{2}+b_{\mid k}^{k}+\frac{1}{2} \Phi^{2} \omega^{2}+\Lambda\right) \bar{g}_{i j}-\frac{1}{2}\left(b_{i \mid j}+b_{j \mid i}\right) \\
& -b_{i} b_{j}+\Phi^{-2}\left\{(\Theta-\Psi) \Theta_{i j}+\Theta_{i j \mid 0}\right. \\
& \left.-\left(\Theta_{\mid 0}-\Psi \Theta+\frac{2}{3} \Theta^{2}+\frac{1}{2} \sigma^{2}\right) \bar{g}_{i j}\right\}=8 \pi \mathbf{G}\left(p \bar{g}_{i j}\right. \\
& \left.+\pi_{i j}\right) .
\end{aligned}
$$

The equations from (89) will be called the spatial Einstein field equations (SEFE). Next, we take $X=\partial / \partial x^{0}$ and $Y=\delta / \delta x^{i}$ in (88) and, by using (73a) and (84), we deduce that

$$
\begin{aligned}
& \bar{R}_{i 0 k}^{k}+\Theta b_{i}-\Theta_{i k} b^{k}-\Phi^{2} \omega_{i k}=-8 \pi \mathrm{G} \Phi q_{i}, \\
& \Theta_{i \mid k}^{k}-\Theta_{\mid i}+\Theta c_{i}-\Theta_{i k} c^{k}-\Phi^{2}\left(\omega_{i \mid k}^{k}+c_{k} \omega_{i}^{k}-2 \omega_{i k} b^{k}\right) \\
& \quad=-8 \pi \mathrm{G} \Phi q_{i} .
\end{aligned}
$$

The equations from either (90a) or (90b) are called mixed Einstein field equations (MEFE). Finally, take $X=Y=\partial / \partial x^{0}$ in (88), and, by using (73c), (2a), and (81), we infer that

$$
\Phi^{2} \overline{\mathbf{R}}+\frac{2}{3} \Theta^{2}-\sigma^{2}+\Phi^{4} \omega^{2}=2 \Phi^{2}(\Lambda+8 \pi \mathbf{G} \rho) .
$$

We call (91) the temporal Einstein field equation (TEFE). Thus Einstein field equations (88) are splitting into three groups of equations given by (89), (90a), (90b), and (91). It is worth mentioning that these equations are expressed in terms of spatial tensor fields and their covariant derivatives induced by the Riemannian spatial connection.

Next, by contracting (89) by $\bar{g}^{i j}$ and using (74) we deduce that the spatial scalar curvature is given by

$$
\begin{aligned}
\frac{1}{2} \overline{\mathbf{R}}= & 2\left(b^{2}+b_{\mid k}^{k}\right)+\frac{3}{2} \Phi^{2} \omega^{2}+3 \Lambda \\
& -\Phi^{-2}\left\{\Theta^{2}+\Theta_{\mid 0}+\frac{3}{2} \sigma^{2}-2 \Psi \Theta\right\}-24 \pi \mathbf{G} p
\end{aligned}
$$

Comparing (92) with (91), we obtain the Raychaudhuri-Ehlers equation induced by the general $(1+3)$ threading of the spacetime:

$$
\begin{aligned}
\Theta_{\mid 0}+ & \frac{1}{3} \Theta^{2}+\sigma^{2}-\Psi \Theta \\
& -\Phi^{2}\left\{b^{2}+b_{\mid k}^{k}+\Phi^{2} \omega^{2}+\Lambda-4 \pi G(\rho+3 p)\right\} \\
= & 0 .
\end{aligned}
$$

Note that (93) is the generalization of (6.4) from [4], which was obtained for the particular case $\Phi=1$.

\section{A New Splitting of Conservation Laws with respect to a General $(1+3)$ Threading of Spacetime}

As is well known, the energy-momentum conservation equations are given by the vanishing of the divergence of $T$. In order to obtain their explicit form, we consider an orthonormal frame field $\left\{E_{k}, U=\Phi^{-1} \partial / \partial x^{0}\right\}$, and according to $[7$, p. 86$]$ we have

$$
(\operatorname{div} T)(X)=\sum_{k=1}^{3}\left(\nabla_{E_{k}} T\right)\left(E_{k}, X\right)-\left(\nabla_{U} T\right)(U, X)=0,
$$

for all $X \in \Gamma(\mathrm{TM})$. Then, take $X=\partial / \partial x^{0}$, in (94), and by using (B.1) and (B.2) obtain

$$
\begin{aligned}
& \Phi^{2}\left\{T_{0 \mid j}^{j}+\left(2 b^{j}-c^{j}\right) T_{j 0}-\Theta^{j k} T_{j k}\right\}-\frac{\partial T_{00}}{\partial x^{0}} \\
& +(2 \Psi-\Theta) T_{00}=0 .
\end{aligned}
$$

Similarly, take $X=\delta / \delta x^{i}$ in (94) and by using (B.3) and (B.4) we deduce that

$$
\begin{gathered}
\Phi^{2}\left\{T_{i \mid j}^{j}-\omega_{i j} T_{0}^{j}+T_{i j} b^{j}\right\}+(\Psi-\Theta) T_{i 0}-T_{i 0 \mid 0}+T_{00} b_{i} \\
-\Theta_{i j} T_{0}^{j}=0 .
\end{gathered}
$$


Now, from (84) and (86), we infer that

$$
\begin{aligned}
& T_{0}^{j}=-\Phi q^{j}, \\
& T_{i}^{j}=p \delta_{i}^{j}+\pi_{i}^{j} .
\end{aligned}
$$

Then, by using (24), (81), (84), (86), (97a), and (97b) in (95) and (96) and taking into account that both $\sigma_{i j}$ and $\pi_{i j}$ are trace-free spatial tensor fields, we obtain the energy conservation equation:

$$
\frac{\partial \rho}{\partial x^{0}}+(\rho+p) \Theta+\sigma^{j k} \pi_{j k}+\Phi\left(q_{\mid j}^{j}+2 b_{j} q^{j}\right)=0
$$

and the momentum conservation equation:

$$
\begin{aligned}
q_{i \mid 0}+ & \frac{4}{3} \Theta q_{i}+\left(\sigma_{i j}+\Phi^{2} \omega_{i j}\right) q^{j} \\
& +\Phi\left\{\frac{\delta p}{\delta x^{i}}+\pi_{i \mid j}^{j}+(p+\rho) b_{i}+\pi_{i j} b^{j}\right\}=0 .
\end{aligned}
$$

In order to compare with what is known in the literature with respect to the $(1+3)$ threading of spacetime, we note that

$$
\nabla_{U} U=b^{k} \frac{\delta}{\delta x^{k}}
$$

This is obtained by direct calculations using (23d). Also, we should remark that (100) states that though the velocity $U$ is a timelike vector field, the acceleration $\nabla_{U} U$ is a spatial vector field.

Remark 7. The above conservation laws are obtained in the most general setting. Indeed, if in particular $\Phi=1,(98)$ and (99) become (5.11) and (5.12) from [4, p. 92], respectively. If, moreover, we have a perfect fluid on $M$, that is, $q_{i}=0$ and $\pi_{i j}=0$, then (98) and (99) become (1) and (2) from Proposition 5 in [7, p. 339].

\section{Splitting of Einstein Field Equations in an Almost FLRW Universe}

Let $M$ be an FLRW universe, whose line element is given by

$$
d \widetilde{s}^{2}=\widetilde{g}_{a b} d x^{a} d x^{b}=a^{2}(\tau)\left\{-d \tau^{2}+\delta_{i j} d x^{i} d x^{j}\right\},
$$

where $\tau$ is the conformal time on $M$ and the threedimensional space given by $\tau=$ const. is an Euclidean space. The Lorentz metric given by (101) is called the background metric. For a more realistic model of the universe, there has been studied perturbation of this metric (cf. $[4,8,9])$. The line element of the full metric $g$ on $M$ is expressed as follows:

$$
d s^{2}=g_{a b} d x^{a} d x^{b}=\tilde{g}_{a b} d x^{a} d x^{b}+\delta g_{a b} d x^{a} d x^{b},
$$

where $\delta g_{a b}$ determine the perturbation. In the present paper we consider the conformal-Newtonian gauge case, for which the full metric is given by

$$
d s^{2}=a^{2}(\tau)\left\{-(1+2 A) d \tau^{2}+(1-2 B) \delta_{i j} d x^{i} d x^{j}\right\},
$$

where $A$ and $B$ are the well-known Bardeen invariants. The spacetime $(M, g)$ with $g$ given by (103) is called an almost FLRW universe.

Now, by using (2b) for (103), we deduce that $\xi_{i}=0, i \in$ $\{1,2,3\}$, which imply

$$
\begin{aligned}
\frac{\delta}{\delta x^{i}} & =\frac{\partial}{\partial x^{i}}, \\
\omega_{i j} & =0, \\
a_{i} & =0, \\
\bar{g}_{i j} & =a^{2}(1-2 B) \delta_{i j}, \\
\bar{g}^{i j} & =\frac{1}{a^{2}(1-2 B)} \delta^{i j} .
\end{aligned}
$$

Also, according to the notation in (2a), we have

$$
\Phi^{2}=a^{2}(1+2 A) .
$$

From (104b) we see that the spatial distribution SM of $(M, g)$ is integrable, but its leaves are not anymore Euclidean spaces. Moreover, by using (12a), (12b), (6d), (104d), and (105), we obtain

$$
\begin{aligned}
\Theta_{i j} & =a^{2}\left\{(1-2 B) \mathscr{H}-B^{\prime}\right\} \delta_{i j}, \\
\Theta & =3\left\{\mathscr{H}-\frac{B^{\prime}}{1-2 B}\right\}, \\
\Psi & =\mathscr{H}+\frac{A^{\prime}}{1+2 A},
\end{aligned}
$$

where "|" denotes derivative with respect to $\tau$ and $\mathscr{H}=$ $a^{\prime} / a$ is the Hubble parameter of the background metric. By calculations, using (106a), (106b), and (104d) into (12c), we infer that

$$
\begin{aligned}
\sigma_{i j} & =0, \quad \forall i, j \in\{1,2,3\} . \\
\Theta_{i j} & =\frac{1}{3} \Theta \bar{g}_{i j}, \quad \forall i, j \in\{1,2,3\} .
\end{aligned}
$$

Taking into account that $\Phi^{-2} \Theta_{i j}$ are the local components of the second fundamental form of the leaves of SM (see (23a)) and using (107a) and (107b), we can state the following result on the kinematic quantities and geometry of $(M, g)$.

Theorem 8. Let $(M, g)$ be an almost FLRW universe. Then one has the following assertions:

(i) $(M, g)$ is both vorticity-free and shear-free spacetime.

(ii) The leaves of the spatial distribution are totally umbilical hypersurfaces of $(M, g)$ with mean curvature vector

$$
H=\frac{1}{3} \Phi^{-2} \Theta \frac{\partial}{\partial \tau}
$$


Next, we assume that the energy-momentum tensor for the almost FLRW universe $(M, g)$ takes the perfect fluid form, that is,

$$
\begin{aligned}
q_{i} & =0, \\
\pi_{i j} & =0,
\end{aligned}
$$

$$
\forall i, j \in\{1,2,3\}
$$

Then, the Bardeen invariants coincide (cf. [4, p. 259]); that is, from now on in our calculations we put $A$ instead of $B$. First, by using (24), (104c), (6b), (105), and (104e), we deduce that

$$
\begin{aligned}
b_{i} & =c_{i}=\frac{A_{i}}{1+2 A}, \\
b^{2} & =\frac{1}{a^{2}\left(1-4 A^{2}\right)(1+2 A)} \sum_{k=1}^{3}\left(A_{k}\right)^{2},
\end{aligned}
$$

where we put $A_{i}=\partial A / \partial x^{i}$. The local coefficients of the spatial Riemannian connection $\bar{\nabla}$ are given by (see (20a), (20b), (C.1), (14), (107a), and (107b))

$$
\begin{aligned}
\bar{\Gamma}_{i}^{k}{ }_{j} & =\frac{1}{1-2 A}\left\{\delta_{i j} A^{k}-\delta_{i}^{k} A_{j}-\delta_{j}^{k} A_{i}\right\}, \\
K_{i}^{j} & =\Theta_{i}^{j}=\frac{1}{3} \Theta \delta_{i}^{j},
\end{aligned}
$$

where we have $A^{k}=A_{k}$. Then, by using (110a) and (111a), we obtain the spatial covariant derivative and the divergence of the acceleration, given by

$$
\begin{aligned}
b_{i \mid j} & =\frac{1}{1+2 A}\left\{A_{i j}+\frac{8 A}{1-4 A^{2}} A_{i} A_{j}\right. \\
& \left.-\frac{1}{1-2 A} \delta_{i j} \sum_{k=1}^{3}\left(A_{k}\right)^{2}\right\}, \\
b_{\mid k}^{k} & =\frac{1}{a^{2}\left(1-4 A^{2}\right)} \sum_{k=1}^{3}\left\{A_{k k}+\frac{2 A-3}{1-4 A^{2}}\left(A_{k}\right)^{2}\right\},
\end{aligned}
$$

where we put

$$
A_{i j}=\frac{\partial^{2} A}{\partial x^{i} \partial x^{j}} .
$$

Now, by direct calculation using (106b), we infer that

$$
\begin{aligned}
& \Theta_{\mid 0}=\frac{\partial \Theta}{\partial \tau}=3\left(\mathscr{H}^{\prime}-\frac{2\left(A^{\prime}\right)^{2}+(1-2 A) A^{\prime \prime}}{(1-2 A)^{2}}\right), \\
& \Theta_{\mid i}=\frac{\partial \Theta}{\partial x^{i}}=-\frac{3}{(1-2 A)^{2}}\left\{2 A^{\prime} A_{i}+(1-2 A) A_{i}^{\prime}\right\} .
\end{aligned}
$$

Also, by using (107b), (111b), (114a), and (114b) and taking into account that $\bar{\nabla}$ is a metric connection, we obtain

$$
\begin{aligned}
\Theta_{i j \mid 0} & =\frac{1}{3} \Theta_{\mid 0} \bar{g}_{i j}=\frac{a^{2}}{1-2 A}\left\{(1-2 \mathrm{~A})^{2} \mathscr{H}^{\prime}\right. \\
- & \left.(1-2 A) A^{\prime \prime}-2\left(A^{\prime}\right)^{2}\right\} \delta_{i j}, \\
\Theta_{i \mid k}^{k} & =\frac{1}{3} \Theta_{\mid i}=-\frac{1}{(1-2 A)^{2}}\left\{2 A^{\prime} A_{i}+(1-2 A) A_{i}^{\prime}\right\} .
\end{aligned}
$$

Finally, by using (C.7) and (C.8) in (74), we deduce that the $3 \mathrm{D}$ Einstein gravitational tensor field is given by

$$
\begin{aligned}
\bar{G}_{i j} & =\frac{1}{(1-2 A)^{2}}\left\{3 A_{i} A_{j}+(1-2 A) A_{i j}\right. \\
& \left.-\delta_{i j} \sum_{k=1}^{3}\left(2\left(A_{k}\right)^{2}+(1-2 A) A_{k k}\right)\right\} .
\end{aligned}
$$

Now, we are in a position to present the splitting of EFE for the almost universe $(M, g)$. First, we consider that the background energy-momentum tensor $\widetilde{T}$ takes the perfect fluid form; that is, we have

$$
\begin{aligned}
\widetilde{T}_{i j} & =\widetilde{p} \widetilde{g}_{i j}=\tilde{p} a^{2} \delta_{i j}, \\
\widetilde{T}_{i o} & =0, \\
\widetilde{T}_{00} & =a^{2} \widetilde{\rho},
\end{aligned}
$$

where $\widetilde{\rho}$ and $\widetilde{p}$ are the relativistic density and pressure, respectively. Then, the energy-momentum tensor $T$ of the perturbed universe $(M, g)$ should have the perfect fluid form too. Hence, by (86), (84), and (81), we have

$$
\begin{aligned}
& T_{i j}=p \bar{g}_{i j}=p a^{2}(1-2 A) \delta_{i j}, \\
& T_{i o}=0, \\
& T_{00}=\Phi^{2} \rho=a^{2}(1+2 A) \rho,
\end{aligned}
$$

where we put

$$
\begin{aligned}
& p=\widetilde{p}+\delta p, \\
& \rho=\widetilde{\rho}+\delta \rho .
\end{aligned}
$$

After some long calculations using (116), (110a), (110b), (112a), (112b), (114a), (106a), (106b), (106c), (107a), (107b), (104d), 
(105), and (118a) into (89), we deduce that the SEFE for the almost FLRW universe $(M, g)$ are given by

$$
\begin{aligned}
& \frac{2}{1-4 A^{2}}\left\{2 A A_{i j}+\frac{1+4 A+12 A^{2}}{1-4 A^{2}} A_{i} A_{j}\right\} \\
& +\delta_{i j}\left\{\frac { 1 } { 1 + 2 A } \left(2 A^{\prime \prime}+\frac{6+4 A}{1+2 A} \mathscr{H} A^{\prime}\right.\right. \\
& \left.+\frac{6 A-1}{1-4 A^{2}}\left(A^{\prime}\right)^{2}-(1-2 A)\left(\mathscr{H}^{2}+2 \mathscr{H}^{\prime}\right)\right) \\
& -\frac{1}{1-4 A^{2}}\left(4 A \sum_{k=1}^{3} A_{k k}\right. \\
& \left.\left.+\frac{3+4 A+12 A^{2}}{1-4 A^{2}} \sum_{k=1}^{3}\left(A_{k}\right)^{2}\right)\right\}=(8 \pi \mathrm{G} p-\Lambda) \\
& \cdot a^{2}(1-2 A) \delta_{i j} .
\end{aligned}
$$

Next, since $(M, g)$ is vorticity-free and $q_{i}=0,(90 \mathrm{~b})$ becomes

$$
\Theta_{\mid i}-\Theta c_{i}=0
$$

via (107b) and (115b). Then, by using (106b), (110a), and (114b) into (121), we obtain the following $\operatorname{MEFE}$ for $(M, g)$ :

$$
\begin{aligned}
(1 & +6 A) A^{\prime} A_{i}+\left(1-4 A^{2}\right) A_{i}^{\prime}+(1-2 A)^{2} \mathscr{H} A_{i} \\
& =0 .
\end{aligned}
$$

Also, by using (C.8), (106b), and (105) into (91) and taking into account that $\sigma^{2}=\omega^{2}=0$, we deduce that the TEFE for $(M, g)$ is given by

$$
\begin{gathered}
\frac{1+2 A}{(1-2 A)^{3}} \sum_{k=1}^{3}\left\{3\left(A_{k}\right)^{2}+2(1-2 A) A_{k k}\right\} \\
\quad+3\left(\mathscr{H}-\frac{A^{\prime}}{1-2 A}\right)^{2} \\
=a^{2}(1+2 A)(\Lambda+8 \pi \mathbf{G} \rho) .
\end{gathered}
$$

Finally, Raychaudhuri-Ehlers equation (93) becomes

$$
\begin{aligned}
& 3\left\{\mathscr{H}^{\prime}-\frac{1}{1-2 A} A^{\prime \prime}-\frac{1}{1-4 A^{2}} \mathscr{H} A^{\prime}\right. \\
& \left.\quad-\frac{4 A}{(1-2 A)\left(1-4 A^{2}\right)}\left(A^{\prime}\right)^{2}\right\}-\frac{1}{1-2 A}\left\{\sum_{k=1}^{3} A_{k k}\right. \\
& \left.\quad-\frac{2}{1-4 A^{2}} \sum_{k=1}^{3}\left(A_{k}\right)^{2}\right\}=a^{2}(1+2 A)(\Lambda \\
& \quad-4 \pi \mathbf{G}(\rho+3 p)) .
\end{aligned}
$$

Summing up the above results, we state the following theorem.
Theorem 9. Let $(M, g)$ be an almost FLRW universe with the energy-momentum tensor of a perfect fluid. Then one has the following assertions:

(i) The SEFE, MEFE, and TEFE of $(M, g)$ are given by (120), (122), and (123), respectively.

(ii) The Raychaudhuri-Ehlers equation in $(M, g)$ is given by (124).

In particular, suppose that $A=0$ and $\Lambda=0$. Then, (123) and (124) become

$$
\begin{aligned}
& \mathscr{H}^{2}=\frac{8 \pi \mathbf{G}}{3} a^{2} \rho, \\
& \mathscr{H}^{\prime}=-\frac{4 \pi \mathbf{G}}{3} a^{2}(\rho+3 p),
\end{aligned}
$$

respectively. Note that, in this case, (122) is trivial, and (120) is a consequence of (125) and (9.24). As (125) and (9.24) are the well-known Friedmann equations for an FLRW universe (cf. (8.4) and (8.5) in [8]), we are entitled to call (123) and (124) the perturbed Friedmann equations in an almost FLRW universe.

\section{Conclusions}

The idea to develop $(1+3)$ threading of a spacetime with respect to a nonnormalized vector field came up as a need for the study of the spacetimes whose metrics have the general form of $(2 \mathrm{a}),(2 \mathrm{~b})$, and $(2 \mathrm{c})$ with $\Phi^{2} \neq 1$. We only mention here all the metrics from both the theory of cosmological perturbations and the theory of black holes.

The main difference between our approach and the methods developed in earlier papers consists in the fact that we deal with the spatial tensor fields as intrinsic objects from the geometry of the spatial distribution. In earlier literature, the spatial tensor fields have been considered as projections on the spatial distribution of tensor fields defined on $M$. This was the main obstacle in defining a correct spatial covariant differentiation for the general case when the spatial distribution is not integrable (cf. (4.19) of [4]). In particular, if the spatial distribution is integrable, the ADM formalism (cf. [3]) can be applied. However, even in this case, the threading formalism is totally different from the ADM formalism. This is because in the ADM formalism the frame field is used,

$$
\left\{\frac{\partial}{\partial x^{i}}, \frac{\delta}{\delta x^{0}}=\frac{\partial}{\partial x^{0}}-N^{i} \frac{\partial}{\partial x^{i}}\right\},
$$

which is adapted to the $3 D$ spatial foliation. Thus, all the formulas like (89), (90a), (90b), (91), (98), and (99) from our paper are expressed with respect to the frame field (126). The advantages of the threading formalism of spacetime with respect to an arbitrary timelike vector field are the following:

(i) It can be applied to any Lorentz metric given by (2a), (2b), and (2c) with $\Phi^{2} \neq 1$. In particular, the perturbation theory and the black holes theory are suitable for this formalism. 
(ii) The spatial distribution is not supposed to be necessarily integrable, and therefore this formalism can be used in a study of any cosmological model.

(iii) It provides, for the first time in literature, a spatial covariant differentiation for spacetimes with nonzero vorticity.

The approach we develop in the paper is based on spatial tensor fields and on the Riemannian spatial connection which behave as $3 D$ geometric objects with local components defined on a $4 D$ spacetime. It is noteworthy that the three groups of EFE presented in the paper are expressed in terms of spatial tensor fields and their covariant derivatives induced by the Riemannian spatial connection. This enables us to write down a splitting of EFE for an almost FLRW universe, which might have an important role in the difficult task of finding models for such a universe. Moreover, the approach can be extended to the study of threading of higher-dimensional universes. This can be seen in a paper of the first author on the threading of a $5 D$ universe (cf. [2]).

\section{Appendix}

\section{A. Details of Formulas of Section 4}

We shall present here details about the formulas stated in Section 4. For the curvature tensor $R$ of the Levi-Civita connection $\nabla$ on $(M, g)$, we use the formula

$$
R(X, Y) Z=\nabla_{X} \nabla_{Y} Z-\nabla_{Y} \nabla_{X} Z-\nabla_{[X, Y]} Z,
$$

for all $X, Y, Z \in \Gamma(\mathrm{TM})$. First, by using (23a), (6b), and (23c), we deduce that

$$
\begin{aligned}
\nabla_{\delta / \delta x^{k}} & \nabla_{\delta / \delta x^{j}} \frac{\delta}{\delta x^{i}}=\nabla_{\delta / \delta x^{k}}\left(\bar{\Gamma}_{i k}^{h} \frac{\delta}{\delta x^{h}}+\Phi^{-2} K_{l k} \frac{\partial}{\partial x^{0}}\right) \\
= & \frac{\delta \bar{\Gamma}_{i}^{h} j}{\delta x^{k}} \frac{\delta}{\delta x^{h}}+\bar{\Gamma}_{i j}^{l}\left(\bar{\Gamma}_{l k}^{h} \frac{\delta}{\delta x^{h}}+\Phi^{-2} K_{l k} \frac{\partial}{\partial x^{0}}\right) \\
& -2 \Phi^{-2} K_{i j} c_{k} \frac{\partial}{\partial x^{0}}+\Phi^{-2} \frac{\delta K_{i j}}{\delta x^{k}} \frac{\partial}{\partial x^{0}} \\
& +\Phi^{-2} K_{i j}\left(K_{k}^{h} \frac{\delta}{\delta x^{h}}+c_{k} \frac{\partial}{\partial x^{0}}\right) \\
= & \left\{\frac{\delta \bar{\Gamma}_{i}^{h} j}{\delta x^{k}} \frac{\delta}{\delta x^{h}}+\bar{\Gamma}_{i}^{l} \bar{\Gamma}_{l k}^{h}+\Phi^{-2} K_{i j} K_{k}^{h}\right\} \frac{\delta}{\delta x^{h}} \\
& +\Phi^{-2}\left\{\frac{\delta K_{i j}}{\delta x^{k}}+K_{l k} \bar{\Gamma}_{i j}^{l}-K_{i j} c_{k}\right\} \frac{\partial}{\partial x^{0}} .
\end{aligned}
$$

Then, by using (5a) and (23b), we obtain

$$
\nabla_{\left[\delta / \delta x^{k}, \delta / \delta x^{j}\right]} \frac{\delta}{\delta x^{i}}=2 \omega_{j k} K_{i}^{h} \frac{\delta}{\delta x^{h}}+2 b_{i} \omega_{j k} \frac{\partial}{\partial x^{0}} .
$$

Now, taking account of (A.2) and (A.3) in (A.1) and using (27a) and the spatial covariant derivative of the extrinsic tensor field, we infer that

$$
\begin{aligned}
& R\left(\frac{\delta}{\delta x^{k}}, \frac{\delta}{\delta x^{j}}, \frac{\delta}{\delta x^{i}}\right)=\left\{\bar{R}_{i j k}^{h}\right. \\
& \left.+\Phi^{-2}\left(K_{i j} K_{k}^{h}-K_{i k} K_{j}^{h}\right)\right\} \frac{\delta}{\delta x^{h}} \\
& \quad+\left\{\Phi^{-2}\left(K_{i j \mid k}-K_{i k \mid j}+K_{i k} c_{j}-K_{i j} c_{k}\right)\right. \\
& \left.\quad-2 b_{i} \omega_{j k}\right\} \frac{\partial}{\partial x^{0}} .
\end{aligned}
$$

Similar calculations by using (23a), (23b), (23c), (23d), (6d), and (5b) lead us to the following:

$$
\begin{aligned}
& \nabla_{\delta / \delta x^{k}} \nabla_{\partial / \partial x^{0}} \frac{\delta}{\delta x^{i}}\left\{\frac{\delta K_{i}^{h}}{\delta x^{k}}+K_{i}^{j} \bar{\Gamma}_{j k}^{h}+b_{i} K_{k}^{h}\right\} \frac{\delta}{\delta x^{h}} \\
&+\left\{\Phi^{-2} K_{i}^{j} K_{j k}+\frac{\delta b_{i}}{\delta x^{k}}+b_{i} c_{k}\right\} \frac{\partial}{\partial x^{0}}, \\
& \nabla_{\partial / \partial x^{0}} \nabla_{\delta / \delta x^{k}} \frac{\delta}{\delta x^{i}}\left\{\frac{\partial \bar{\Gamma}_{i k}^{h}}{\partial x^{0}}+K_{j}^{h} \bar{\Gamma}_{i k}^{j}+K_{i k} b^{h}\right\} \frac{\delta}{\delta x^{h}} \\
&+\left\{\Phi^{-2} \frac{\partial K_{i k}}{\partial x^{0}}+b_{j} \bar{\Gamma}_{i k}^{j}-\Psi \Phi^{-2} K_{i k}\right\} \frac{\partial}{\partial x^{0}}, \\
& \nabla_{\left[\delta / \delta x^{k}, \partial / \partial x^{0}\right]} \frac{\delta}{\delta x^{i}}=-a_{k} K_{i}^{h} \frac{\delta}{\delta x^{h}}-b_{i} a_{k} \frac{\partial}{\partial x^{0}} .
\end{aligned}
$$

Then, by using (A.5), (A.6), and (A.1) and taking into account (27b) and both covariant derivatives of the extrinsic curvature tensor field, we deduce that

$$
\begin{aligned}
& R\left(\frac{\delta}{\delta x^{k}}, \frac{\partial}{\partial x^{0}}, \frac{\delta}{\delta x^{i}}\right)=\left\{\bar{R}_{i 0 k}^{h}+b_{i} K_{k}^{h}-b^{h} K_{i k}\right\} \frac{\delta}{\delta x^{k}} \\
& \quad+\left\{b_{i \mid k}+b_{i} b_{k}\right. \\
& \left.\quad-\Phi^{-2}\left(K_{i k \mid 0}+K_{i j} K_{k}^{j}-\Psi K_{i k}\right)\right\} \frac{\partial}{\partial x^{0}} .
\end{aligned}
$$

Next, we consider the curvature tensor fields of type $(0,4)$ of the connections $\nabla$ and $\bar{\nabla}$, denoted by $R$ and $\bar{R}$ and given by

$$
\begin{aligned}
R(X, Y, Z, U) & =g(R(X, Y, U), Z), \\
\bar{R}(X, Y, \mathcal{\delta} Z, \mathcal{S U}) & =\bar{g}(\bar{R}(X, Y, \mathcal{S} U), \mathcal{S} Z),
\end{aligned}
$$


for all $X, Y, Z, U \in \Gamma(\mathrm{TM})$. Then we have the following components with respect to the threading frame:

$$
\begin{aligned}
& R_{i l j k}=R\left(\frac{\delta}{\delta x^{k}}, \frac{\delta}{\delta x^{j}}, \frac{\delta}{\delta x^{l}}, \frac{\delta}{\delta x^{i}}\right)=\bar{g}_{l h} R_{i j k}^{h}, \\
& R_{i 0 j k}=R\left(\frac{\delta}{\delta x^{k}}, \frac{\delta}{\delta x^{j}}, \frac{\partial}{\partial x^{0}}, \frac{\delta}{\delta x^{i}}\right)=-\Phi^{2} R_{i j k}^{0}, \\
& R_{i l 0 k}=R\left(\frac{\delta}{\delta x^{k}}, \frac{\partial}{\partial x^{0}}, \frac{\delta}{\delta x^{l}}, \frac{\delta}{\delta x^{i}}\right)=\bar{g}_{l h} R_{i 0 k}^{h}, \\
& R_{i 00 k}=R\left(\frac{\delta}{\delta x^{k}}, \frac{\partial}{\partial x^{0}}, \frac{\partial}{\partial x^{0}}, \frac{\delta}{\delta x^{i}}\right)=-\Phi^{2} R_{i 0 k}^{0}, \\
& \bar{R}_{i l j k}=\bar{R}\left(\frac{\delta}{\delta x^{k}}, \frac{\delta}{\delta x^{j}}, \frac{\delta}{\delta x^{l}}, \frac{\delta}{\delta x^{i}}\right)=\bar{g}_{l h} \bar{R}_{i j k}^{h}, \\
& \bar{R}_{i l 0 k}=\bar{R}\left(\frac{\delta}{\delta x^{k}}, \frac{\partial}{\partial x^{0}}, \frac{\delta}{\delta x^{l}}, \frac{\delta}{\delta x^{i}}\right)=\bar{g}_{l h} \bar{R}_{i 0 k}^{h} .
\end{aligned}
$$

These local components are used in both Sections 4 and 5 in order to deduce the final form for the structure equations and the local components of the Ricci tensor, respectively.

\section{B. Details of Formulas of Section 8}

We derive some useful formulas for the conservation equations stated in Section 8. First, by using (80), (23d), and (83a), we obtain

$$
\begin{aligned}
& \left(\nabla_{U} T\right)\left(U, \frac{\partial}{\partial x^{0}}\right)=\left(\nabla_{\Phi^{-1}\left(\partial / \partial x^{0}\right)} T\right)\left(\Phi^{-1} \frac{\partial}{\partial x^{0}}, \frac{\partial}{\partial x^{0}}\right) \\
& =\Phi^{-2}\left\{\frac{\partial T_{00}}{\partial x^{0}}-2 T\left(\nabla_{\partial / \partial x^{0}} \frac{\partial}{\partial x^{0}}, \frac{\partial}{\partial x^{0}}\right)\right\} \\
& =\Phi^{-2}\left\{\frac{\partial T_{00}}{\partial x^{0}}-2 T\left(\Phi^{2} b^{k} \frac{\delta}{\delta x^{k}}+\Psi \frac{\partial}{\partial x^{0}}, \frac{\partial}{\partial x^{0}}\right)\right\} \\
& =\Phi^{-2}\left\{\frac{\partial T_{00}}{\partial x^{0}}-2 \Psi T_{00}\right\}-2 b^{j} T_{j 0} .
\end{aligned}
$$

Then, by using (59), (83a), (60), (23a), (80), and (85a), we deduce that

$$
\begin{aligned}
& \sum_{k=1}^{3}\left\{\left(\nabla_{E_{k}} T\right)\left(E_{k}, \frac{\partial}{\partial x^{0}}\right)\right\} \\
& =\sum_{k=1}^{3}\left\{\left(\nabla_{E_{k}^{h}\left(\delta / \delta x^{h}\right)} T\right)\left(E_{k}^{j} \frac{\delta}{\delta x^{j}}, \frac{\partial}{\partial x^{0}}\right)\right\} \\
& =\sum_{k=1}^{3}\left\{E_{k}^{h} \frac{\delta}{\delta x^{h}}\left(E_{k}^{j} T_{j 0}\right)\right. \\
& -E_{k}^{h} T\left(\nabla_{\delta / \delta x^{h}}\left(E_{k}^{j} \frac{\delta}{\delta x^{j}}\right), \frac{\partial}{\partial x^{0}}\right)
\end{aligned}
$$

$$
\begin{aligned}
& \left.-E_{k}^{h} E_{k}^{j} T\left(\frac{\delta}{\delta x^{j}}, \nabla_{\delta / \delta x^{h}} \frac{\partial}{\partial x^{0}}\right)\right\}=\bar{g}^{j h}\left\{\frac{\delta T_{j 0}}{\delta x^{h}}\right. \\
& -T\left(\bar{\Gamma}_{j}^{k} \frac{\delta}{h x^{k}}+\left(\omega_{j h}+\Phi^{-2} \Theta_{j h}\right) \frac{\partial}{\partial x^{0}}, \frac{\partial}{\partial x^{0}}\right) \\
& \left.-T\left(\frac{\delta}{\delta x^{j}},\left(\Theta_{h}^{k}+\Phi^{2} \omega_{h}^{k}\right) \frac{\delta}{\delta x^{k}}+c_{k} \frac{\partial}{\partial x^{0}}\right)\right\}=T_{0 \mid j}^{j} \\
& -\Phi^{-2} \Theta T_{00}-\Theta^{j k} T_{j k}-c^{j} T_{j 0} .
\end{aligned}
$$

Next, by similar calculations, we infer that

$$
\begin{aligned}
& \left(\nabla_{U} T\right)\left(U, \frac{\delta}{\delta x^{i}}\right) \\
& =\Phi^{-2}\left\{T_{i 0 \mid 0}-\Psi T_{i 0}-b_{i} T_{00}\right\}-T_{i j} b^{j}, \\
& \sum_{k=1}^{3}\left\{\left(\nabla_{E_{k}} T\right)\left(E_{k}, \frac{\delta}{\delta x^{i}}\right)\right\} \\
& =T_{i \mid j}^{j}-\Phi^{-2} \Theta T_{i 0}-\left(\omega_{i j}+\Phi^{-2} \Theta_{i j}\right) T_{0}^{j} .
\end{aligned}
$$

\section{Ricci Tensor Calculations}

In this appendix we present the calculations for both the spatial Ricci tensor and the spatial scalar curvature of an almost FLRW universe. First, by using (21), (104a), (104d), and (104e), we obtain

$$
\bar{\Gamma}_{i}^{k}{ }_{j}=\frac{1}{1-2 B}\left\{\delta_{i j} B^{k}-\delta_{i}^{k} B_{j}-\delta_{j}^{k} B_{i}\right\},
$$

where we put $B^{k}=B_{k}=\partial B / \partial x^{k}$. Then (20a) and (C.1) imply

$$
\bar{g}\left(\bar{\nabla}_{\partial / \partial x^{j}} \frac{\partial}{\partial x^{i}}, \frac{\partial}{\partial x^{h}}\right)=a^{2}\left\{\delta_{i j} B_{h}-\delta_{i h} B_{j}-\delta_{j h} B_{i}\right\} .
$$

Then, apply $\partial / \partial x^{k}$ to both sides in (C.2) and, taking into account that $\bar{\nabla}$ is a metric connection, we deduce that

$$
\begin{aligned}
& \bar{g}(\left.\bar{\nabla}_{\partial / \partial x^{k}} \bar{\nabla}_{\partial / \partial x^{j}} \frac{\partial}{\partial x^{i}}, \frac{\partial}{\partial x^{h}}\right) \\
&+\bar{g}\left(\bar{\nabla}_{\partial / \partial x^{j}} \frac{\partial}{\partial x^{i}}, \bar{\nabla}_{\partial / \partial x^{k}} \frac{\partial}{\partial x^{h}}\right) \\
&=a^{2}\left\{\delta_{i j} B_{h k}-\delta_{i h} B_{j k}-\delta_{j h} B_{i k}\right\},
\end{aligned}
$$

where we put

$$
B_{j k}=\frac{\partial^{2} B}{\partial x^{j} \partial x^{k}} .
$$

Denote the left hand side of (C.3) by $L_{i h j k}$ and by using (25), (26a), (20a), and (C.1) we infer that

$$
\begin{gathered}
L_{i h j k}-L_{i h k j}=R_{i j k}^{l} \bar{g}_{l h}+\left(\bar{\Gamma}_{i j}^{l} \bar{\Gamma}_{h k}^{m}-\bar{\Gamma}_{i k}^{l} \bar{\Gamma}_{k}^{m}{ }_{j}^{m}\right) \bar{g}_{l m} \\
=\bar{R}_{i h j k}+\frac{a^{2}}{1-2 B}\left\{\left(\delta_{i j} B^{l}-\delta_{i}^{l} B_{j}-\delta_{j}^{l} B_{i}\right)\right.
\end{gathered}
$$




$$
\begin{aligned}
& \cdot\left(\delta_{h k} B_{l}-\delta_{h l} B_{k}-\delta_{k l} B_{h}\right)-\left(\delta_{i k} B^{l}-\delta_{i}^{l} B_{k}-\delta_{k}^{l} B_{i}\right) \\
& \left.\cdot\left(\delta_{h j} B_{l}-\delta_{h l} B_{j}-\delta_{j l} B_{h}\right)\right\} .
\end{aligned}
$$

Thus, (C.3) and (C.5) imply

$$
\begin{gathered}
\bar{R}_{i h j k}=a^{2}\left\{\delta_{i j} B_{h k}+\delta_{h k} B_{i j}-\delta_{i k} B_{h j}-\delta_{j h} B_{i k}\right\} \\
+\frac{a^{2}}{1-2 B}\left\{\left(\delta_{i k} b^{l}-\delta_{i}^{l} B_{k}-\delta_{k}^{l} B_{i}\right)\right. \\
\cdot\left(\delta_{h j} B_{l}-\delta_{h l} B_{j}-\delta_{j l} B_{h}\right)-\left(\delta_{i j} b^{l}-\delta_{i}^{l} B_{j}-\delta_{j}^{l} B_{i}\right) \\
\left.\cdot\left(\delta_{h k} B_{l}-\delta_{h l} B_{k}-\delta_{k l} B_{h}\right)\right\} .
\end{gathered}
$$

Now, contracting (C.6) by $\bar{g}^{h k}$ and using (67), (70), and (104e), we obtain

$$
\begin{aligned}
\bar{R}_{i j} & =\frac{1}{(1-2 B)^{2}}\left\{\delta_{i j} \sum_{k=1}^{3}\left(B_{k}\right)^{2}+3 B_{i} B_{j}\right. \\
& \left.+(1-2 B)\left(B_{i j}+\delta_{i j} \sum_{k=1}^{3} B_{k k}\right)\right\}, \\
\overline{\mathbf{R}} & =\frac{2}{a^{2}(1-2 B)^{3}}\left\{3 \sum_{k=1}^{3}\left(B_{k}\right)^{2}+2(1-2 B) \sum_{k=1}^{3} B_{k k}\right\} .
\end{aligned}
$$

\section{Competing Interests}

The authors declare that there is no conflict of interests regarding the publication of this paper.

\section{References}

[1] A. Bejancu and C. Călin, "On the $(1+3)$ threading of spacetime with respect to an arbitrary timelike vector field," The European Physical Journal C, vol. 75, article 159, 2015.

[2] A. Bejancu, "Kinematic quantities and Raychaudhuri equations in a 5D universe," The European Physical Journal C, vol. 75, article 346, 2015.

[3] C. W. Misner, K. S. Thorne, and J. A. Wheeler, Gravitation, W. H. Freeman, New York, NY, USA, 1997.

[4] G. F. R. Ellis, R. Maartens, and M. A. H. MacCallum, Relativistic Cosmology, Cambridge University Press, Cambridge, UK, 2012.

[5] A. Zelmanov, Chronometric Invariants, American Research Press, Rehoboth, Mass, USA, 2006.

[6] S. Kobayashi and K. Nomizu, Foundations of Differential Geometry, vol. 1, Interscience Publishers, New York, NY, USA, 1963.

[7] B. O’Neill, Semi-Riemannian Geometry and Applications to Relativity, Academic Press, New York, NY, USA, 1983.

[8] K. A. Malik and D. Wands, "Cosmological perturbations," Physics Reports, vol. 475, no. 1-4, pp. 1-51, 2009.

[9] V. F. Mukhanov, H. A. Feldman, and R. H. Brandenberger, "Theory of cosmological perturbations," Physics Reports, vol. 215, no. 5-6, pp. 203-333, 1992. 


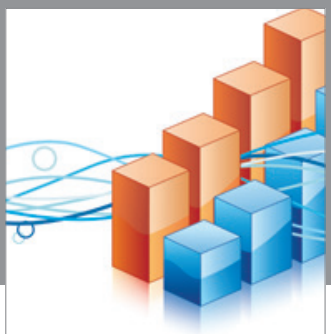

Advances in

Operations Research

vatem alat4

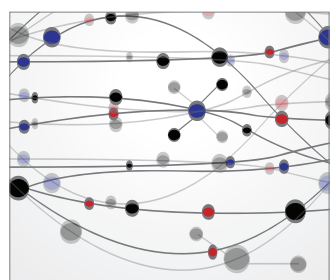

\section{The Scientific} World Journal
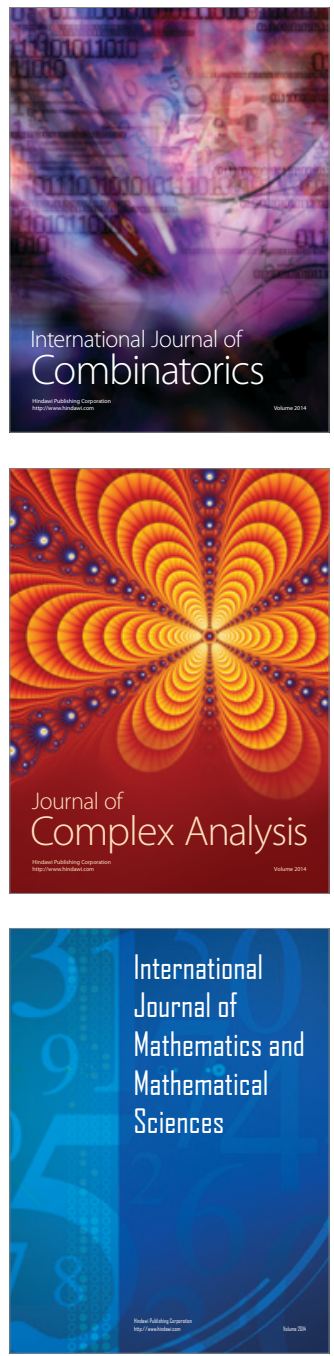
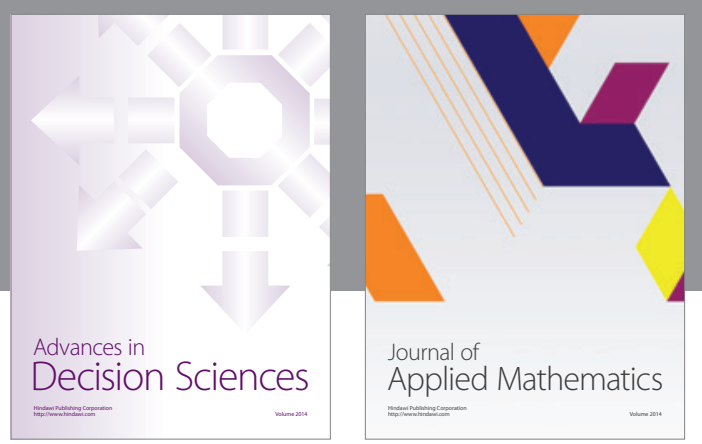

Algebra

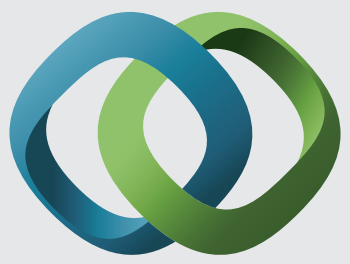

\section{Hindawi}

Submit your manuscripts at

http://www.hindawi.com
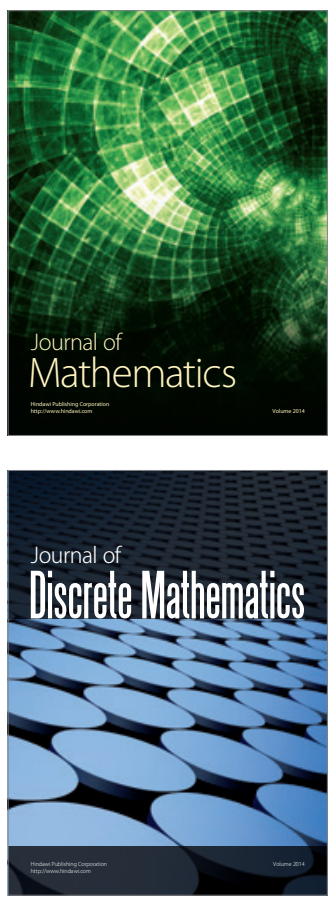

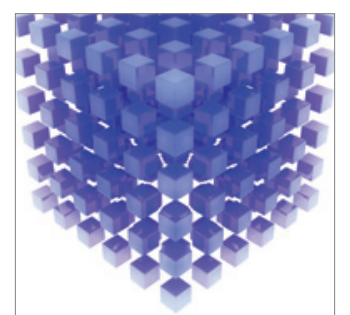

Mathematical Problems in Engineering
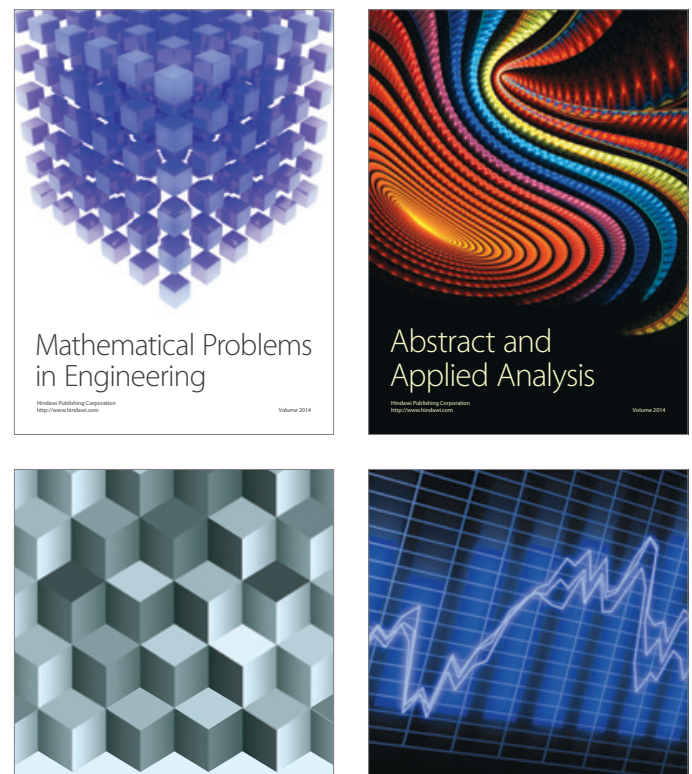

Journal of

Function Spaces

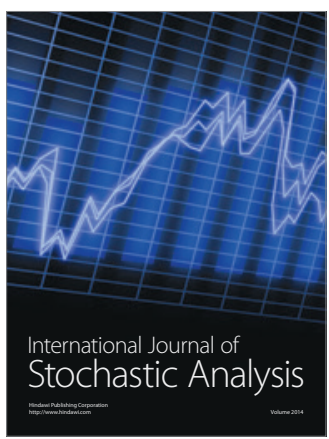

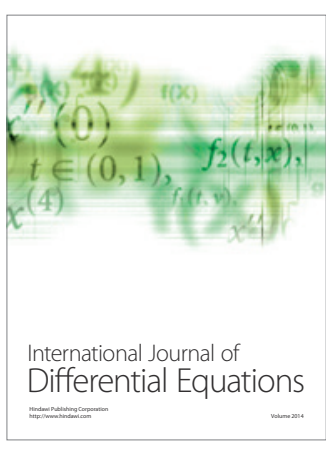
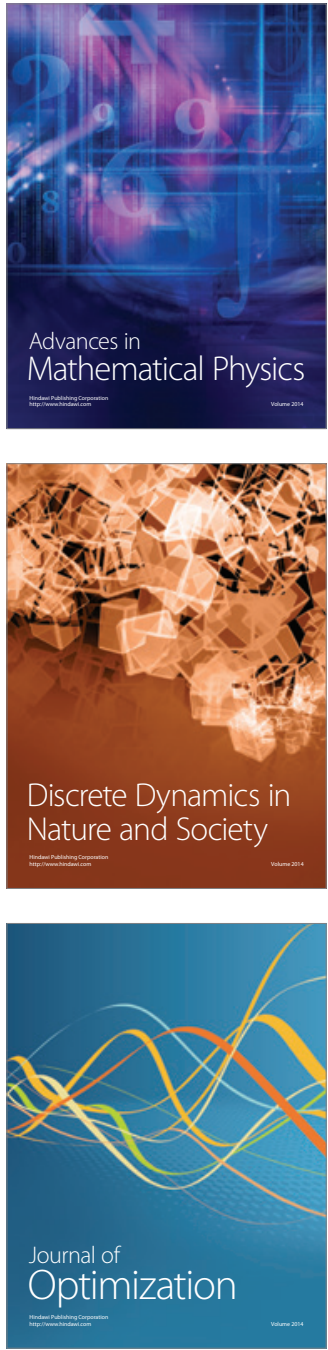